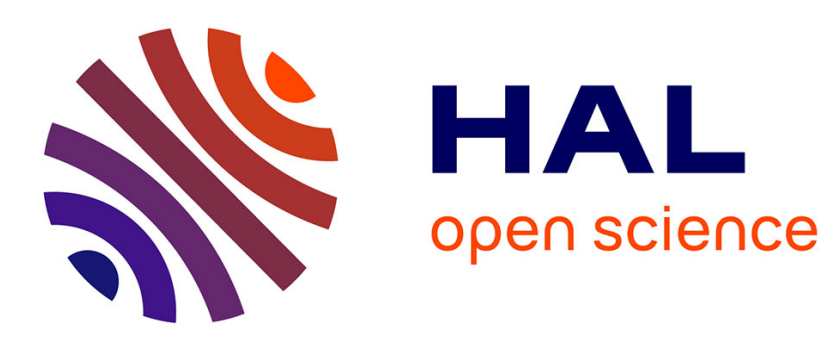

\title{
Non-dispersal and density properties of infinite packings
}

Alexandre Delyon, Antoine Henrot, Yannick Privat

\section{To cite this version:}

Alexandre Delyon, Antoine Henrot, Yannick Privat. Non-dispersal and density properties of infinite packings. SIAM Journal on Control and Optimization, 2019, 57 (2), pp.1467-1492. 10.1137/18M1181183. hal-01753911v2

\section{HAL Id: hal-01753911 \\ https://hal.science/hal-01753911v2}

Submitted on 5 Jun 2019

HAL is a multi-disciplinary open access archive for the deposit and dissemination of scientific research documents, whether they are published or not. The documents may come from teaching and research institutions in France or abroad, or from public or private research centers.
L'archive ouverte pluridisciplinaire HAL, est destinée au dépôt et à la diffusion de documents scientifiques de niveau recherche, publiés ou non, émanant des établissements d'enseignement et de recherche français ou étrangers, des laboratoires publics ou privés. 


\title{
NON-DISPERSAL AND DENSITY PROPERTIES OF INFINITE PACKINGS
}

\author{
ALEXANDRE DELYON*, ANTOINE HENROT ${ }^{\dagger}$, AND YANNICK PRIVAT ${ }^{\ddagger}$
}

\begin{abstract}
This article is motivated by an optimization problem arising in Biology. Interpreting the eggs arrangements (packings) in the brood chamber as results from an optimization process, we are led to look for packings that are at the same time the most possible dense and non-dispersed. We first model this issue in terms of an elementary shape optimization problem among convex bodies, involving their inradius, diameter and area. We then solve it completely, showing that the solutions are either particular hexagons or a symmetric 2-cap body, namely the convex hull of a disk and two points lined-up with the center of the disk.
\end{abstract}

Key words. shape optimization, tiling domains, density of packings.

AMS subject classifications. 52A40, 52A10, 49K30, 49Q10.

1. Introduction. This article is devoted to investigating optimal configurations of infinite packings in the two-dimensional space $\mathbb{R}^{2}$. Recall that a packing associated to a convex body $K$ with non-empty interior is an arrangement of non-overlapping copies of $K$. More precisely, denoting by $\mathcal{K}$ the set of compact convex bodies of $\mathbb{R}^{2}$, an infinite packing $P(K)$ with pattern $K$ is defined by

$$
P(K)=\bigcup_{i \in I} \tau_{i}(K)
$$

where $I$ denotes a countable set of indices, and the mappings $\tau_{i}$ are affine isometries of $\mathbb{R}^{2}$ such that $\operatorname{int}\left(\tau_{i}(K)\right) \cap \operatorname{int}\left(\tau_{j}(K)\right)=\emptyset$ for all $i \neq j$.

Since we are interested in infinite packings, we will consider without loss of generality in what follows that $I=\mathbb{N}$ and we will denote by $\mathcal{P}(K)$ the set of all infinite packings of the plane with pattern $K$.

A close notion that will be much discussed in the sequel is the one of tiling domains. Recall that, as a consequence of the definition of packings, a convex $K$ defines a tiling domain of the plane whenever $\mathbb{R}^{2} \in \mathcal{P}(K)$.

In the whole article, the notation $|\cdot|$ will denote the Lebesgue measure in $\mathbb{R}^{2}$.

Let us make precise the shape optimization problem we will deal with. The criterion to minimize involves two geometrical functionals denoted $d$ and $D_{\infty}$. Let us define them.

- The first one models the density of a packing. We choose to define it as follows, see Section 2.1 for a discussion and the link with another classical quantity for the density.

$$
d(K)=\frac{|K|}{\left|K^{T}\right|}
$$

for every convex set $K$, where $K^{T}$ denotes the smallest convex set tiling the plane and containing $K$ (we refer to Appendix A for the proof that such a set

\footnotetext{
${ }^{*}$ Université de Lorraine, CNRS, Institut Elie Cartan de Lorraine, BP 7023954506 Vandœuvrelès-Nancy Cedex, France (alexandre.delyon@univ-lorraine.fr)

${ }^{\dagger}$ Université de Lorraine, CNRS, Institut Elie Cartan de Lorraine, BP 7023954506 Vandœuvrelès-Nancy Cedex, France (antoine.henrot@univ-lorraine.fr).

${ }^{\ddagger}$ IRMA, Université de Strasbourg, CNRS UMR 7501, 7 rue René Descartes, 67084 Strasbourg, France (yannick.privat@unistra.fr).
} 
exists). In some sense, the quantity $d(K)$ stands for a quantitative measure of the tiling ability of $K$. Roughly speaking, we can consider that the highest $d(K)$ is and the most tilling will be the convex set $K$. Notice in particular that if $K$ is tiling, then $d(K)=1$.

- The second functional is defined by

$$
D_{\infty}(K)=\frac{2 \sqrt{|K|}}{\sqrt{\pi} \operatorname{Diam}(K)}
$$

for every convex set $K$, where $\operatorname{Diam}(K)$ denotes the diameter of $K$. As this will be highlighted in the sequel, the quantity $D_{\infty}(K)$ is a measure of nondispersal of any packing associated to the convex $K$. Indeed, this quantity is obtained by introducing the restriction of a packing with pattern $K$ to a disk with diameter $R>0$, by comparing the diameter of this set with the diameter of the disk, and by letting $R$ tend to $+\infty$. Hence, trying to minimize $D_{\infty}(K)$ will allow to obtain a convex $K$ and an associated packing as "compact" as possible.

Note that modelling issues and in particular the functionals choices will be discussed and commented in Section 2.1.

Finally, for a given $t \in[0,1]$, we will consider in the sequel a convex combination of both previous criteria. The resulting criterion, denoted $J_{t}$ reads

$$
J_{t}(K)=t d(K)+(1-t) \frac{1}{D_{\infty}(K)} .
$$

Let us define the admissible set. We will deal with three kinds of constraints:

(i) the considered sets will be compact and convex subsets of $\mathbb{R}^{2}$,

(ii) to avoid that the shapes collapse, we impose to the considered convex sets to have a minimal inradius $r_{0}$. In what follows, we will denote by $r(K)$ the inradius of any convex set $K$.

(iii) since the functionals we will deal with are invariant by homothety, it is relevant to assume the area of the pattern prescribed, equal to a positive constant $A$.

We now introduce the complete shape optimization problem we will solve.

Let $t \in[0,1], r_{0}>0$ and $A \geqslant \pi r_{0}^{2}$ be fixed and let $\mathcal{A}_{r_{0}, A}$ denote the set of compact convex sets having an inradius larger than $r_{0}$ and an area equal to $A$, namely

$$
\mathcal{A}_{r_{0}, A}=\left\{K \in \mathcal{K} \mid r(K) \geqslant r_{0} \text { and }|K|=A\right\} .
$$

The shape optimization problem we will consider reads

$$
\sup _{K \in \mathcal{A}_{r_{0}, A}} J_{t}(K) .
$$

It is notable that this problem is also motivated by applied considerations. Some explanations about the biological framework in which this problem naturally arises are provided at the end of this section.

Let us roughly state hereafter the main results of this article. More detailed (and technical) versions of these theorems are provided in Section 2.2

Our first result deals with generalities about tiling domains. It seems to us interesting in its own. 
Theorem A. Among all (convex) tiling sets with given diameter and inradius, the one of minimal area is a p-hexagon, in other words a hexagon with two parallel opposite sides with same length. By duality, one shows that the (convex) tiling set with given area and inradius maximizing its diameter is a p-hexagon.

Our second result deals with the solution of Problem (1.3). For the sake of clarity, we state it informally.

THEOREM B. Under a smallness assumption on the ratio $r_{0}^{2} / A$, the solutions of Problem (1.3) are either a p-hexagon or a symmetric 2-cap body (the convex hull of a disk and two points lined-up with the center of the disk), depending on the values of the parameter $t$.

Complete and extensive versions of these results are provided in Theorems 2.7 and 2.9.

We end this section by giving an interpretation of this problem in Biology. The shape optimization problem (1.3) is related to the understanding of the eggs shape of a class of crustaceans, subclass branchiopoda, called eulimnadia.

In a clutch, the eggs are placed in the brood chamber, which is located dorsally beneath the carapace and which is closed by the abdominal processes. To understand eggs geometry, it appears relevant to interpret the observed arrangements as the result of an optimization process. This way, assuming that resulting shapes allow to the crustacean to incubate the largest number of eggs, we look for configurations guaranteeing at the same time that shapes and arrangements make the resulting packing the most "dense" (this word meaning here the "most tiling", see the definition of $d(K))$ and the most "compact" (in the sense that the restriction of the packing to a given ball with large radius will contain the largest number of elements). In a nutshell, denoting by $K$ the egg shape and assuming that the clutch contains the largest possible number of eggs, it is plausible that eggs arrangements look at maximizing at the same time $d(K)$ and $D_{\infty}(K)$. We formalize this idea by looking for patterns $K$ maximizing a convex combination of these functionals, whence the writing of Problem (1.3).

Structure of the article. This article is organized as follows. Section 2.1 is devoted to several remarks about our motivations for considering Problem (1.3), as well as our functional and admissible set choices. The main results of this article are gathered in Section 2.2. Section 3 is devoted to the proof of Theorem 2.7, whereas Section 4 is devoted to the proof of Theorem 2.9.

1.1. Notations. Hereafter, we sum-up the notations we will use throughout this article.

$\mathcal{K} \quad$ set of compact convex bodies of $\mathbb{R}^{2}$

$D(0, R) \quad$ centered closed ball with radius $R$

$|K| \quad$ area of a convex body $K$

$r(K) \quad$ inradius of a convex body $K$

$\operatorname{Diam}(K)$ diameter of a convex body $K$

$H_{r}^{*} \quad$ regular hexagon with inradius $r$

2. Modelling and solving the optimization problem.

2.1. Modelling issues and state of the art.

Density of convex sets. Let $K \in \mathcal{K}$ and $P(K)$ be a packing with pattern $K$. It is standard (see [12]) to define the density of $\delta(P(K))$ as

$$
\delta(P(K))=\liminf _{r \rightarrow+\infty} \frac{\sharp\left\{i \in \mathbb{N} \mid \tau_{i}(K) \subset[-r / 2, r / 2]^{2}\right\}|K|}{r^{2}} .
$$


For a fixed $r>0$, the ratio $\sharp\left\{i \in \mathbb{N} \mid \tau_{i \in I}(K) \subset[-r / 2, r / 2]^{2}\right\}|K| / r^{2}$ represents the rate of the area occupied by the elements of the packing $P(K)$ contained in $[-r / 2, r / 2]^{2}$ with respect to the total area of a square with side $r$. Letting $r \rightarrow+\infty$ makes this definition independent of the window in which this rate is evaluated.

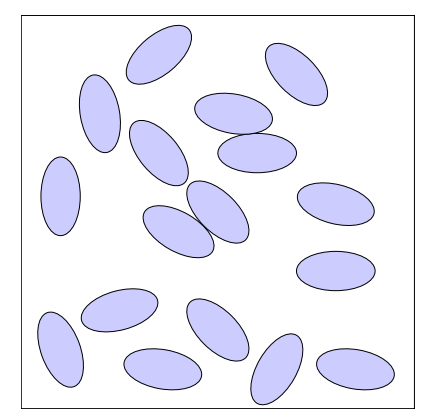

FIG. 1. An example of a packing with ellipses, we can see the density as the ratio between the blue area and the entire square

Having in mind to look for packings maximizing (among other criteria) the density functional, it is relevant to introduce a criterion depending only on the pattern choice, by setting $d_{1}(K)=\sup _{P(K) \in \mathcal{P}(K)} \delta(P(K))$, corresponding to the optimal density of a packing associated to the pattern $K$. This quantity is called density of the convex $K[12]$.

Notice that the following elementary properties about $d_{1}$ are direct consequences of the definition.

Proposition 2.1. For every $K \in \mathcal{K}$, one has $d_{1}(K) \in[0,1]$. Moreover,

1. if $D$ is a disk, $d_{1}(D)=\frac{\pi}{2 \sqrt{3}} \simeq 0.9$ [5].

2. if $K$ is a tiling domain, $d_{1}(K)=1$.

3. if $K \in \mathcal{K}$ and $T \in \mathcal{K}$ is tiling such that $K \subset T$ then $d_{1}(K) \geqslant \frac{|K|}{|T|}[10]$.

The last property will be crucial in the sequel, since it allows to provide a lower bound for $d_{1}$. Roughly speaking, the main ingredient consists in considering a tilling domain $T$ such that $K \subset T$, the family of sets $\left\{\tau_{i}(T)\right\}_{i \in \mathbb{N}}$ defining the associated packing. We then define a packing with pattern $K$ by placing a copy of $K$ in each cell $\tau_{i}(T)$, and to observe that the density of this packing will be larger than $|K| /|T|$. Moreover, it has been shown that given a convex body $K$, there exists a triangle $T$ such that $K \subset T$ and $|K| /|T| \geqslant 2 / 3$ (see [4] by M.A Fary in 1950 and [2] by R.Courant in 1965). By considering parallelograms instead of triangles, Kuperberg obtained in 1982 in [10] the same conclusion, and this way the lower bound $d_{1}(K) \geqslant 3 / 4$ for every convex body $K$. This lower bound has been improved in 1990 by W. and G. Kuperberg in [9], where it is shown that $d_{1}(K) \geqslant \sqrt{3} / 2$, by using a particular tiling hexagon. In 1995, K.R Doheny proved in [3] the existence of $r_{0}>\sqrt{3} / 2$ such that $d_{1}(K) \geqslant r_{0}$ for every convex body $K$. Up to our knowledge, the exact value of the bound $\inf \left\{d_{1}(K), K \in \mathcal{K}\right\}$ remains unknown. 


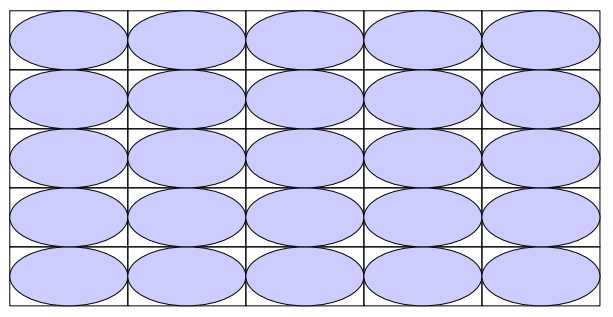

FIG. 2. A packing with ellipses in a tiling with rectangles. It is intuitive that the density of this packing is equal to the ratio of the area of the ellipse over the area of the rectangle

Unfortunately, the precise value of $d_{1}(K)$ is almost never computable, even for simple choices of $K$. More annoying, having in mind to consider it as criterion of an optimization problem, the quantity $d_{1}(K)$ appears intricate to handle. These considerations lead us to consider as an alternative and more workable definition of the density the functional $d$ defined by (1.1) involving the smallest convex tiling domain containing $K$. Obviously there holds $d(K) \leqslant d_{1}(K)$ for every convex body $K$ and it is notable that all the properties gathered in Proposition 2.1 above remain satisfied with this new definition of density.

Non-dispersal properties of convex sets. Let us first model the notion of nondispersion for packings. We start from the observation that balls are the "less dispersed" bodies, in the sense that, among all nonempty convex sets, they minimize the ratio of the diameter by the square-root of their area. This leads us to define the notion of "non-dispersion" of packings by comparing their diameter to the one of balls. More, precisely, we introduce, mimicking the definition of $\delta$ in (2.1):

$$
D_{\infty}^{\prime}(K)=\inf _{P \in \mathcal{P}(K)} \limsup _{R \rightarrow \infty} \frac{2 R}{\sqrt{\sharp\left\{i, \tau_{i}(K) \subset D(0, R)\right\}} \operatorname{Diam}(K)},
$$

the lim sup being used in the definition to make $D_{\infty}^{\prime}(K)$ independent of the balls radii. More precisely, given a packing $P \in \mathcal{P}(K)$ and $R>0$, we consider a disk with radius $R$ and evaluate the number of copies of $K$ within the disk. Note also that we take the square root of this integer in the definition by observing that the maximal number of identical copies of a convex order of magnitude in a disk with radius $R$ is ${ }^{1} \mathrm{O}\left(R^{2}\right)$. Finally, the diameter of $K$ appearing in the denominator is used as a renormalization factor. This appears natural in view of defining an adimensional quantity.

First an elementary reasoning shows that, in a disk of radius $R$, there cannot be more than $\pi R^{2} /|K|$ copies of $K$. As a consequence, we infer that

$$
D_{\infty}^{\prime}(K) \geqslant \frac{2 \sqrt{|K|}}{\sqrt{\pi} \operatorname{Diam}(K)}=D_{\infty}(K)
$$

for every $K \in \mathcal{K}$, where $D_{\infty}(K)$ is defined by (1.2). The following result, whose proof is postponed to Appendix $\mathrm{B}$, provides fine estimates of $D_{\infty}^{\prime}(K)$.

\footnotetext{
${ }^{1}$ Indeed, let us provide a sketch of argument. Let us consider a rectangle tiling the plane and containing the convex body. We denote by $L$ and $\ell$ its dimensions. If $R>>L$, the number of rectangles that can be packed within a disk with radius $R$ is $\mathrm{O}\left(\pi R^{2}\right) /(L \ell)=\mathrm{O}\left(R^{2}\right)$. Therefore, the number of copies of a convex $K$ that can be packed within a disk is less than $\pi R^{2} /|K|=\mathrm{O}\left(R^{2}\right)$
} 

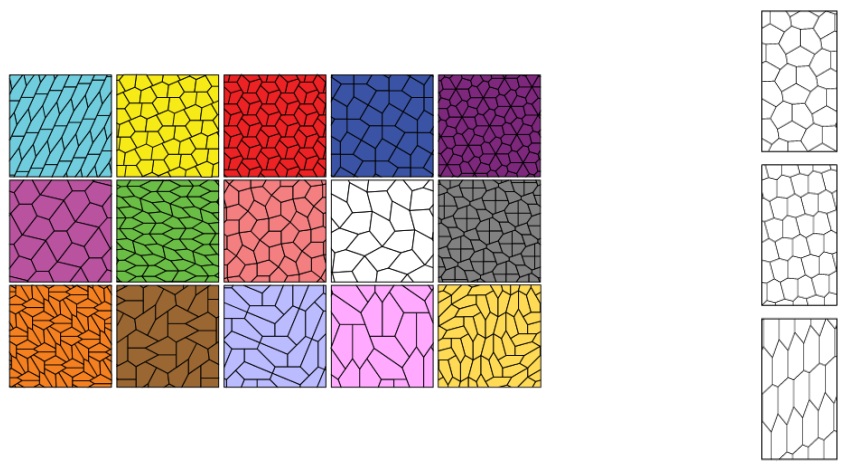

FIG. 3. (Left) The fifteen kinds of tiling pentagons (Source https://commons.wikimedia.org/ wiki/File:PentagonTilings15.svg). (Right) The three kinds of tiling hexagon (Source http:// mathworld.wolfram.com/HexagonTiling.html)

Theorem 2.2. Let $K \in \mathcal{K}$. One has

$$
\frac{2 \sqrt{|K|}}{\sqrt{\pi} \operatorname{Diam}(K)} \leqslant D_{\infty}^{\prime}(K) \leqslant \sqrt{\frac{2}{\sqrt{3}}} \frac{2 \sqrt{|K|}}{\sqrt{\pi} \operatorname{Diam}(K)} .
$$

Furthermore, if $K$ is tiling, then one has $D_{\infty}^{\prime}(K)=D_{\infty}(K)$.

According to the result above, one has $D_{\infty}^{\prime}(K) / D_{\infty}(K) \in[1,1.08)$. We infer that, in order to consider workable quantities, it will be relevant in the sequel to consider $D_{\infty}$ as criterion of non-dispersal.

Convex tiling domains. The previous remarks suggest that we take a short interest about convex tiling domains. Notice that a convexity argument allows to show that a two-dimensional convex domain which is tiling in $\mathbb{R}^{2}$ is necessarily a polygon. More precisely, thanks to Euler's formulae, it is known that a polygon with more than six vertices cannot be tiling [1]. Moreover, any triangle or quadrilateral tiles the plane, but there exist only three kinds of tiling hexagons. The case of pentagons is more intricate. It has been recently solved in [11], by leading an exhaustive search of all families of convex pentagons tiling the plane. In particular, the authors state that there are no more than fifteen kinds of pentagons tiling the plane.

\subsection{Solving the optimization problems.}

Notations. Let us define particular convex sets that will play a crucial role in the sequel.

Definition 2.3 (The hexagons $H_{A, r}$ and $H^{D, r}$ ). Let $r>0$ and $A \geqslant 2 \sqrt{3} r^{2}$. Let $\mathcal{C}$ be a circle centered at the origin $O$ with radius $r$ and $H_{A, r}$ be the hexagon defined as follows:

(i) each side of $H_{A, r}$ is tangent to $\mathcal{C}$.

(ii) Denoting by $\left\{B_{i}\right\}_{i=1, \ldots, 6}$ the set of tangential points ordered between $H_{A, r}$ and $\mathcal{C}$ and by $\theta_{i}$ the angle $\widehat{B_{i} \widehat{O B_{i}+1}}$ (with the convention that $B_{7}=B_{1}$ ), one has

$$
\left\{\begin{array}{l}
\theta_{1}=\theta_{4}=4 \arctan \left(\frac{2 r^{2}+\sqrt{A^{2}-12 r^{4}}}{4 r^{2}+A}\right) \\
\theta_{2}=\theta_{3}=\theta_{5}=\theta_{6}=\frac{\pi-\theta_{1}}{2}
\end{array}\right.
$$

It is notable that $H_{A, r}$ is a a p-hexagon, in other words a hexagon with two parallel opposite sides with same length (see Fig 4). 
Moreover, let $D$ and $r$ be two positive numbers. Noting that one has ${ }^{2}$

$$
\operatorname{Diam}\left(H_{A, r}\right)=\frac{1}{3 r}\left(2 A+\sqrt{A^{2}-12 r^{4}}\right),
$$

one defines the hexagon $H^{D, r}$ by $H^{D, r}=H_{A(D), r}$, where $A(D)$ is the unique solution of the equation

$$
D=\alpha(A(D), r) \quad \text { with } \quad \alpha(A, r)=\frac{1}{3 r}\left(2 A+\sqrt{A^{2}-12 r^{4}}\right) .
$$

Furthermore, one has $\left|H^{D, r}\right|=2 r D-r \sqrt{D^{2}-4 r^{2}}$ (see Appendix C).

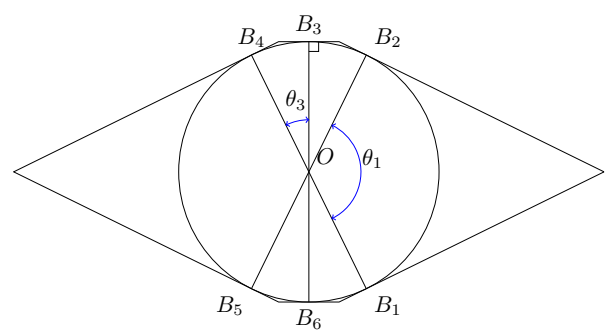

FIG. 4. The p-hexagon $H_{A, r}$ and its inscribed circle.

Definition 2.4 (The symmetric 2-cap bodies $G^{D, r}$ and $G_{A, r}$ ). Let $D$ and $r$ be two positive numbers such that $D \geqslant 2 r$. We denote by $G^{D, r}$ the convex hull of a circle with radius $r$ and two points at a distance of $D$, lined up with the circle center (see Fig. 5). Such a convex set will be called symmetric 2-cap body of diameter $D$ and inradius $r$.

Similarly let $A$ and $r$ be two positive numbers. One defines the symmetric 2-cap body $G_{A, r}$ by $G_{A, r}=G^{D(A), r}$, where $D(A)$ is the unique positive solution of

$$
A=r\left(\sqrt{D(A)^{2}-4 r^{2}}+2 r \arcsin \left(\frac{2 r}{D(A)}\right)\right) .
$$

REMARK 2.5. Let $A, D, r$, be three positive numbers. In [8], it is shown that for every convex set with area $A$, inradius $r$ and diameter $D$, one has

$$
A \geqslant r\left(\sqrt{D^{2}-4 r^{2}}+2 r \arcsin \left(\frac{2 r}{D}\right)\right)
$$

and this inequality is an equality if, and only if $K=G^{D, r}$ (and thus, $A=\left|G^{D, r}\right|$ ). This inequality can also be interpreted as follows: the convex set with diameter $D$ and inradius $r$ having the lowest area is $G^{D, r}$. By duality, this also means that the convex set with area $A$ and inradius $r$ having the maximal diameter is the convex hull of a circle with radius $r$ and two points, lined up with the circle center

\footnotetext{
${ }^{2}$ We refer to Appendix C for a proof of this claim.
} 

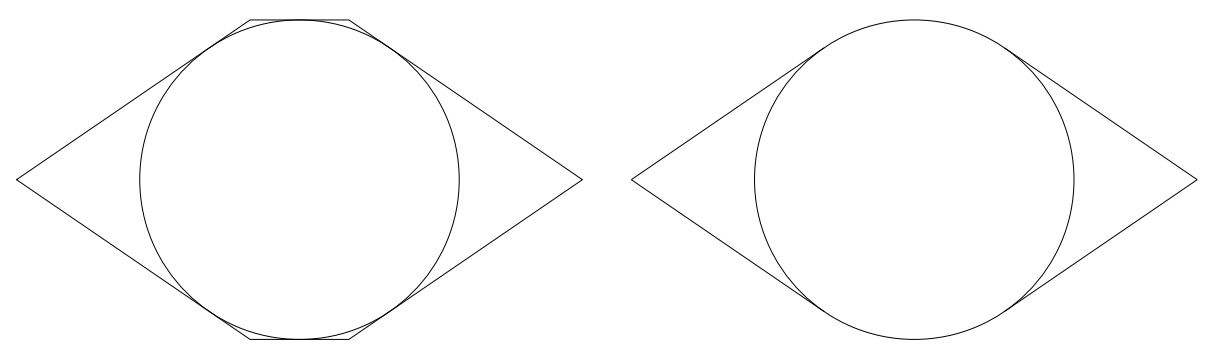

FIG. 5. Left: the hexagon $H_{A, r}$ and its inscribed circle. Right: the symmetric 2-cap body $G^{D, r}$ and its inscribed circle.

REMARK 2.6. It follows easily from geometrical observations or simple computations that

- there exists a unique hexagon (up to rotations) fulfilling the conditions of Definition 2.3, and this construction can be led if, and only if $A \geqslant 2 \sqrt{3} r^{2}$;

- the hexagon $H_{A, r}$ is of area $A$ and inradius $r$;

- the sides of $H_{A, r}$ are two by two parallels. In particular, $H_{A, r}$ is a p-hexagon (see Theorem A for the definition).

- the diameter of $H^{D, r}$ can differ from D. For instance, it is the case if $r^{2} \geqslant A / 2 \sqrt{3}$ and $D \leqslant \min \left\{4 / \sqrt{3} r, \operatorname{Diam}\left(G_{A, r}\right)\right.$, as noted in the proof of Lemma 4.2.

Statement of the main results. In the following theorem, we state several sharp inequalities for tiling domains of the plane. These results constitute key ingredients of the proof of Theorem 2.9 .

THEOREM 2.7. Let $T$ be a compact convex tiling domain of $\mathbb{R}^{2}$

1. There holds

$$
|T| \geqslant 2 \sqrt{3} r(T)^{2} \quad \text { and } \quad \operatorname{Diam}(T) \geqslant \frac{4}{\sqrt{3}} r(T),
$$

with equality if only if $T$ is a regular hexagon.

2. One has

$$
\operatorname{Diam}(T) \leqslant \frac{1}{3 r(T)}\left(2|T|+\sqrt{|T|^{2}-12 r(T)^{4}}\right),
$$

with equality if, and only if $T=H_{A, r}$

REMARK 2.8. Let $r>0$. As a byproduct of Theorem 2.7, using in particular that the mapping $\left[2 \sqrt{3} r^{2},+\infty\right) \ni A \mapsto \alpha(A, r)$ (where $\alpha$ is given by (2.4)) is increasing, we get that

- the (convex) tiling set with diameter $D$ and inradius $r$ minimizing its area is the hexagon $H^{D, r}$;

- the (convex) tiling set with area $A$ and inradius $r$ maximizing its diameter is the hexagon $H_{A, r}$.

The first point comes from the following observation: let $A \geqslant 2 \sqrt{3} r^{2}$ for some $r>$ 0. Then, the map $F_{r}: A \mapsto \alpha(A, r)=\frac{1}{3 r}\left(2 A+\sqrt{A^{2}-12 r^{4}}\right)$ is increasing and defines a bijection from $\left[2 \sqrt{3} r^{2},+\infty\right)$ to $[4 / \sqrt{3},+\infty)$. Its inverse mapping is $F_{r}^{-1}$ : $[4 / \sqrt{3},+\infty) \ni D \mapsto 2 r D-r \sqrt{D^{2}-4 r^{2}}$.

Now, let $T$ be a tiling domain of $\mathbb{R}^{2}$. According to the considerations above, the inequality (2.6) is equivalent to $\operatorname{Diam}(T) \leqslant F_{r(T)}(|T|)$, which rewrites $F_{r}^{-1}(\operatorname{Diam}(T)) \leqslant$ 
$|T|$. This shows that the inequality

$$
2 r(T) \operatorname{Diam}(T)-r(T) \sqrt{\operatorname{Diam}(T)^{2}-4 r(T)^{2}} \leqslant|T|
$$

holds true for every tiling domain of $\mathbb{R}^{2}$. The expected conclusion follows.

The second claim is a direct consequence of (2.6) in Theorem 2.\%.

THEOREM 2.9. Let $r_{0}$ and $A$ be two positive numbers such that $2 \sqrt{3} r_{0}^{2}<A$.

Let us denote by $X_{0}(\simeq 3.1847)$ the unique zero of the function $X \mapsto \sqrt{X^{2}-4}(14-$ $\left.5 X^{2}\right)+4 X\left(X^{2}-3\right)$ on $[4 / \sqrt{3},+\infty)$ and set

$$
t_{A, r_{0}}=\frac{\sqrt{\pi} /(2 \sqrt{A})}{\sqrt{\pi} /(2 \sqrt{A})+A \gamma_{0} / r_{0}^{3}} \in(0,1) .
$$

with

$$
\gamma_{0}=\frac{\left(2 \sqrt{X_{0}^{2}-4}-X_{0}\right)}{\sqrt{X_{0}^{2}-4}\left(2 X_{0}-\sqrt{X_{0}^{2}-4}\right)^{2}} \simeq 0.0472 .
$$

1. If $t \in\left[0, t_{A, r_{0}}\right]$, the symmetric 2-cap body $G_{A, r_{0}}$ solves Problem (1.3);

2. Let us assume moreover that

$$
r_{0} \leqslant \gamma \sqrt{A} \quad \text { where } \quad \gamma=\frac{1}{\sqrt{2 X_{0}-\sqrt{X_{0}^{2}-4}}} \in[0.5069,0.5070]
$$

and define

$$
t_{A, r_{0}}^{*}=\frac{\frac{\sqrt{\pi}}{2 \sqrt{A}}\left(\operatorname{Diam}\left(G_{A, r_{0}}\right)-\operatorname{Diam}\left(H_{A, r_{0}}\right)\right)}{\frac{\sqrt{\pi}}{2 \sqrt{A}}\left(\operatorname{Diam}\left(G_{A, r_{0}}\right)-\operatorname{Diam}\left(H_{A, r_{0}}\right)\right)+A\left(\frac{1}{\left|\operatorname{Diam}\left(G_{A, r_{0}}\right)\right|}-\frac{1}{\left|\operatorname{Diam}\left(H_{A, r_{0}}\right)\right|}\right)}
$$

One has $t_{A, r_{0}}^{*} \geqslant t_{A, r_{0}}$. Moreover, if $t \in\left[0, t_{A, r_{0}}^{*}\right)$, the symmetric 2-cap body $G_{A, r_{0}}$ solves Problem (1.3), and if $t \in\left(t_{A, r_{0}}^{*}, 1\right]$, the p-hexagon $H_{A, r_{0}}$ solves Problem (1.3). If $t=t_{A, r_{0}}^{*}$, the two convex sets $H_{A, r_{0}}$ and $G_{A, r_{0}}$ solve Problem (1.3).

Remark 2.10 (Comment on the assumption (2.10)). The assumption $2 \sqrt{3} r_{0}^{2}<A$ is natural since it is a sufficient and necessary condition for ensuring the existence of the p-hexagon $H_{A, r_{0}}$ (see the first item of Theorem 2.7). Note that writes also $r_{0} \leqslant \hat{\gamma} \sqrt{A}$ with $\hat{\gamma} \simeq 0.5373$.

The assumption (2.10) appears a bit technical (although relevant from an applied point of view). A refined analysis can show that if $r_{0} / \sqrt{A} \in(\gamma, \hat{\gamma})$, there exists $\tilde{t}_{A, r_{0}} \geqslant t_{A, r_{0}}$ such that for $t \geqslant \tilde{t}_{A, r_{0}}$, either the symmetric 2-cap body $G_{A, r_{0}}$ or the p-hexagon $H_{A, r_{0}}$ solves the problem (1.3).

3. Proof of Theorem 2.7. Proving Theorem 2.7 is equivalent to determine the optimal value of the problems

$$
\inf \left\{|K|, K \in \mathcal{T}, r(K) \geqslant r_{0}\right\} \quad \text { and } \inf \left\{\operatorname{Diam}(K), K \in \mathcal{T}, r(K) \geqslant r_{0}\right\},
$$

and

$$
\sup \left\{\operatorname{Diam}(K), K \in \mathcal{T}, r(K) \geqslant r_{0},|K|=A\right\},
$$


where $\mathcal{T}$ denotes the set of tiling domains in $\mathbb{R}^{2}$. In what follows, we will solve a relaxed version of these problem, namely

$$
\inf \left\{|K|, K \in \mathcal{P}_{6}, r(K) \geqslant r_{0}\right\} \quad \text { and } \inf \left\{\operatorname{Diam}(K), K \in \mathcal{P}_{6}, r(K) \geqslant r_{0}\right\},
$$

and

$$
\sup \left\{\operatorname{Diam}(K), K \in \mathcal{P}_{6}, r(K) \geqslant r_{0},|K|=A\right\}
$$

where $\mathcal{P}_{6}$ denotes the set of convex polygons of the plane having at most six sides, and show that the solutions are tiling domains. As a consequence, and since the new admissible set contains the previous one, the optimal values between the problems (3.1) and their relaxed version will coincide.

Before dealing with each problem separately, let us state some preliminary results allowing to reduce the search of optimal domain to a simpler class. The arguments used in Step 1 below hold indifferently for each problem of (3.3).

As a preliminary remark, notice that the two problems of (3.3) have a solution since $\mathcal{P}_{6}$ is compact for the Hausdorff topology and the functionals $K \mapsto|K|, K \mapsto$ $r(K), K \mapsto \operatorname{Diam}(K)$ restricted to convex sets are continuous for this topology, see [7, chapter 2].

Step 1. Restricting the set of admissible domains. The following lemmas are in order.

LEMma 3.1. For any problem of (3.3) and (3.4), there exists a solution $K^{*}$ that is a hexagon. Moreover, regarding the first problem of (3.3) and Problem (3.4), every solution of one of such problems is necessarily a hexagon.

Proof. Let us assume by contradiction that $K^{*}$ has $N$ sides, with $N<6$. Consider two diametral points $D_{1}$ and $D_{2}$ of $K^{*}$ and let $M$ be any vertex of $K^{*}$ different from $D_{1}$ and $D_{2}$. Then, we change $K^{*}$ into $\hat{K}^{*}$ by removing the vertex $M$ and creating two new vertices as follows: we cut $K^{*}$ with a well-chosen hyperplane at a distance of $M$ small enough so that the diameter and the inner radius of $K^{*}$ are not modified.

- Minimizing the area: the area of $\hat{K}^{*}$ is strictly lower than the area of $K^{*}$ which contradicts the optimality of $K^{*}$. The conclusion follows.

- Minimizing the diameter: the diameter of $\hat{K}^{*}$ being equal to the one of $K^{*}$, we infer that it is possible to restrict our search to hexagons.

- Maximizing the diameter: consider the set $t \hat{K}^{*}$ where $t>1$ is chosen in such a way that $\left|t \hat{K}^{*}\right|=\left|K^{*}\right|$. Then, one has $r\left(t \hat{K}^{*}\right)=\operatorname{tr}\left(\hat{K}^{*}\right)=t r_{0}>r_{0}$ and $\operatorname{Diam}\left(t \hat{K}^{*}\right)=t \operatorname{Diam}\left(\hat{K}^{*}\right)>\operatorname{Diam}\left(\hat{K}^{*}\right)$, which contradicts the optimality of $K^{*}$. The conclusion follows.

REMARK 3.2. It will follow from the proof that all the solutions of Problems (3.3) and (3.4) are hexagons.

The proofs of the two next lemmas are exactly similar for each problems of (3.1) and (3.2). Since this last problem is more constrained and in some sense, more intricate, we prove this lemma for the problem of maximizing the diameter. An easy adaptation of the proof below shows the same result for the issue of minimizing the area or the diameter.

Lemma 3.3. Let $K^{*}$ be a solution of any problem of (3.3) and (3.4). Then, necessarily, $r\left(K^{*}\right)=r_{0}$.

Proof. Let $K^{*}$ be a solution of Problem (3.4) and let us assume by contradiction that $r\left(K^{*}\right)>r_{0}$. Since $K^{*}$ is a convex polygon, there exists two vertices $B$ and 
$C$ of $K^{*}$ such that $\operatorname{Diam}\left(K^{*}\right)=B C$. For $t \in[0,1]$ let $\rho_{t}$ be the stretching with ratio $t$ and direction (kept fixed) the axis $(B C)$. Then, one has $\left|\rho_{t}\left(K^{*}\right)\right|=t\left|K^{*}\right|$ and $\operatorname{Diam}\left(\rho_{t}\left(K^{*}\right)\right)=\operatorname{Diam}\left(K^{*}\right)$. Noting that $[0,1] \ni t \mapsto r\left(\rho_{t}\left(K^{*}\right)\right)$ is a continuous increasing function such that $r(0)=0$ and $r(1)=r\left(K^{*}\right)$, consider $r \in\left(r_{0}, r\left(K^{*}\right)\right)$ and $t \in(0,1]$ such that $r\left(\rho_{t}\left(K^{*}\right)\right)=r$. Let $K_{t}$ be the range of $\rho_{t}\left(K^{*}\right)$ by the homothety centered at $O$, the center of the incircle, with scale factor $1 / \sqrt{t}>1$. Hence, one has $\left|K_{t}\right|=\left|K^{*}\right|, \operatorname{Diam}\left(K_{t}\right)=\operatorname{Diam}\left(K^{*}\right) / \sqrt{t}$ and $r\left(K_{t}\right)=r\left(K^{*}\right) / \sqrt{t}>r_{0}$. It follows that $K_{t}$ is a admissible hexagon and moreover $\operatorname{Diam}\left(K_{t}\right)>\operatorname{Diam}\left(K^{*}\right)$. We have then reached a contradiction.

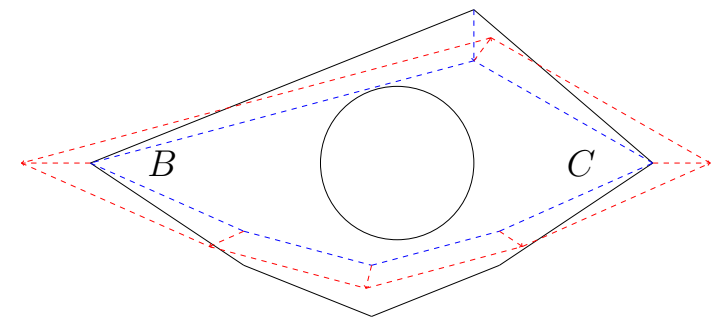

FIG. 6. Illustration of the proof of Lemma 3.3: the hexagon $K^{*}$ (black), the hexagon $\rho_{t}\left(K^{*}\right)$ (blue) and the hexagon $K_{t}$ (red).

Lemma 3.4. Let $K^{*}$ be a solution of any problem of (3.3) and (3.4). Then, necessarily $K^{*}$ is tangent at each side to any inscribed circle.

Proof. We argue by contradiction, by assuming that there exist an inscribed circle $\mathcal{C}$ and a side of $K^{*}$ that does not meet. To reach a contradiction, we will show that one can transform $K^{*}$ into a new admissible set $\hat{K}$ having a strictly larger diameter.

Consider first the case where there exists a side $\left[M M^{\prime}\right]$ at positive distance of $\mathcal{C}$ such that $\operatorname{Diam}\left(K^{*}\right)>M M^{\prime}$. Assume without loss of generality the existence of two vertices of $K^{*}$ different from $M$ and reaching its diameter. This property will allow to construct a new set $\tilde{K}$ from $K^{*}$ by slightly modifying the location of $M$, and such that $\operatorname{Diam}(\tilde{K})=\operatorname{Diam}\left(K^{*}\right)$. Let $N$ be the vertex of $K^{*}$ such that $M$ is adjacent to $N$ and $M^{\prime}$. Let $\lambda \in(0,1)$ and $M_{\lambda}=\lambda N+(1-\lambda) M$. For $\lambda>0$ small enough, there holds $\left(M^{\prime} M_{\lambda}\right) \cap \mathcal{C}=\emptyset$. Hence, denoting by $\tilde{K}$ be the hexagon obtained by replacing $M$ by $M_{\lambda}$, one has $r(\tilde{K})=r\left(K^{*}\right)$. Moreover, since $\tilde{K} \subset K^{*}$ and $\tilde{K} \neq K^{*}$, one has $|\tilde{K}|<\left|K^{*}\right|$. To get $\hat{K}$, we now apply a homothety to $\tilde{K}$ where the scale factor is chosen in such a way that $|\hat{K}|=\left|K^{*}\right|$ (see Fig. 7). We then have $r(\hat{K})>r\left(K^{*}\right)$ and $\operatorname{Diam}(\hat{K})>\operatorname{Diam}\left(K^{*}\right)$, whence the contradiction.

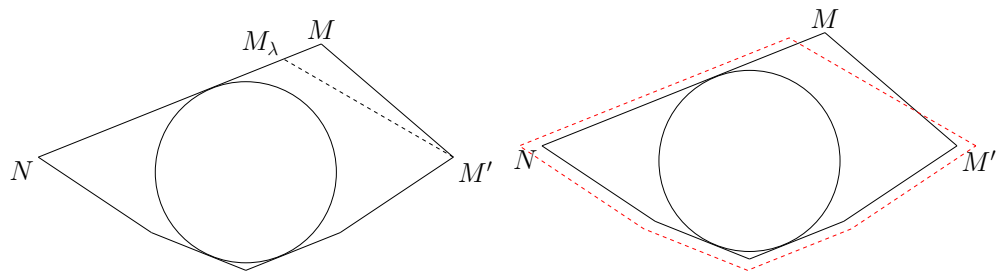

FIG. 7. Geometrical illustration of the method: construction of $\tilde{K}$ (left) and construction of $\hat{K}$ (dotted line) from $\tilde{K}$ (right).

Consider now the complementary case where any side which does not meet tan- 
gentially the circle $\mathcal{C}$ realizes the diameter of $K^{*}$. Hence, let us consider a side $[A B]$ of $K^{*}$ realizing the diameter without meeting $\mathcal{C}$ tangentially. Notice that, if such a choice of side does not exist, then we are in the previous case and we can reach a contradiction.

Denote by $O$ the center of $\mathcal{C}$ and by $M$ the orthogonal projection of $O$ on $(A B)$. Then, $M$ belongs to the segment $[A B]$ and the distance $\delta$ of $M$ to $\mathcal{C}$ is positive (by compactness). The new circle $\mathcal{C}^{\prime}$ obtained from $\mathcal{C}$ by translation of vector $\delta \frac{\overrightarrow{O M}}{O M}$ is tangent to $(A B)$ (see Fig. 8). Let us prove that $\mathcal{C}^{\prime} \subset K^{*}$. Let $(A, \vec{i}, \vec{j})$ be the orthonormal basis such that $\vec{i}=\overrightarrow{A B} / A B$ and $K^{*}$ be contained in $\mathbb{R}_{+}^{2}$. Then, $\partial K^{*} \backslash(A B)$ is parametrized by a positive concave function $f:[0, A B] \mapsto \mathbb{R}_{+}$. For $u \in[0,1]$, let $D_{u}$ be the vertical axis with equation $x=u$. Then, defining $x_{1}=\min \left\{u \in[0,1], D_{u} \cap \mathcal{C} \neq \emptyset\right\}$ and $x_{2}=\max \left\{u \in[0,1], D_{u} \cap \mathcal{C} \neq \emptyset\right\}$, the region $\mathcal{R}=\left\{(x, y), x_{1} \leqslant x \leqslant x_{2}, 0 \leqslant y \leqslant f(x)\right\}$ is contained in $K^{*}$ with an easy convexity argument and by construction, $\mathcal{C}^{\prime} \subset \mathcal{R}$. Hence, $\mathcal{C}^{\prime} \subset K^{*}$ and we are then led to the previous case.

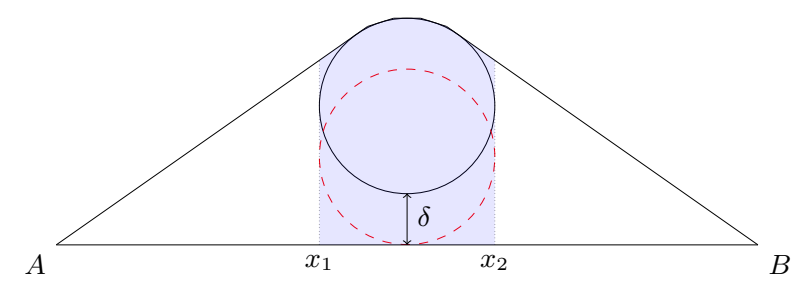

FIG. 8. case where the diameter is realized by the only side $[A B]$ of $K^{*}$ which does not meet $\mathcal{C}$ tangentially.

By combining the three lemmas we have just proved, we will recast both problems of (3.3) in a simpler way by using a convenient parametrization and some analytical arguments. For homogeneity reasons and according to Lemma 3.3, we will assume from now on that $r_{0}=1$, the solutions for the general case being easily inferred from that case.

Let $K^{*}$ be a hexagon solution of a problem of (3.3). Since each problem is invariant under rotation or translation of $K$, we will assume without loss of generality that the center of the inscribed circle (which is uniquely located inside $K^{*}$, according to Lemma 3.4) is the origin $O$ and that one side of $K^{*}$ is included in the axis $x=1$. Let $\left\{B_{i}\right\}_{i=1 . .6}$ be the projections of $O$ on each side of $K^{*}$ with the convention that $B_{1}$ is the projection of $\mathrm{O}$ on the side included in the axis $x=1$, and the other points are located by following the trigonometric sense.

Let $\left\{A_{i}\right\}_{i=1 . . .6}$ be the vertices of $K^{*}$ having positive coordinates in the basis $\left(O ; \overrightarrow{O B_{i}}, \overrightarrow{O B_{i+1}}\right)$, let $\theta_{i}=B_{i} \widehat{O B_{i+1}}$ and $\varphi_{i}={\widehat{B_{i} O A_{i}}}_{i}$, so that

$$
\sum_{i=1}^{6} \theta_{i}=2 \pi \quad \text { and } \quad \sum_{i=1}^{6} \varphi_{i}=\pi
$$

Notice that $0 \leqslant \theta_{i} \leqslant \pi$ and since the two triangles $B_{i} O A_{i}$ and $A_{i} O B_{i+1}$ are similar, one has $\varphi_{i}=\theta_{i} / 2$ (see Fig. 9). 


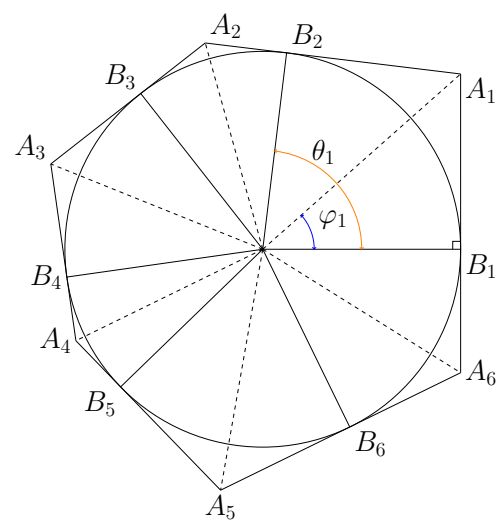

FIG. 9. Parametrization of hexagons

Using this parametrization, let us rewrite each optimization problem in terms of the variables $\varphi_{i}$. Decomposing the hexagon $K^{*}$ into the six quadrilaterals $O B_{i} A_{i} B_{i+1}$ $(i=1, \ldots, 6)$ and each quadrilateral into two similar triangles $B_{i} O A_{i}$ and $B_{i+1} O A_{i}$ (whose area is equal to $\frac{O B_{i} \cdot B_{i} A_{i}}{2}=\frac{\tan \left(\varphi_{i}\right)}{2}$ ), we get

$$
\left|K^{*}\right|=\sum_{i=1}^{6} \tan \varphi_{i}
$$

Introduce the sets $\Theta^{0}=\left\{\Phi=\left(\varphi_{1}, \ldots, \varphi_{6}\right) \in[0, \pi / 2]^{6}, \sum_{i=1}^{6} \varphi_{i}=\pi\right\}$ and $\Theta_{A}=$ $\left\{\Phi \in \Theta^{0}, \sum_{i=1}^{6} \tan \varphi_{i}=A\right\}$. The two problems of (3.3) rewrite

$$
\min _{\Phi \in \Theta^{0}} \sum_{i=1}^{6} \tan \left(\varphi_{i}\right) \quad \text { and } \quad \min _{\Phi \in \Theta^{0}} \operatorname{Diam}(H(\Phi))
$$

whereas Problem (3.4) rewrites

$$
\max _{\Phi \in \Theta_{A}} \operatorname{Diam}(H(\Phi)),
$$

where $H(\Phi)$ denotes the hexagon tangent at each side to the unit circle, whose semi circle center angles are the $\varphi_{i}$ 's.

Step 2. Solving the two problems of (3.5). Let us consider the first problem of (3.5). The proof is straightforward. Indeed, noting that the point-wise constraint $\varphi_{i} \leqslant \pi / 2$ cannot be active, it follows from the Karush-Kuhn-Tucker theorem the existence of a Lagrange multiplier $\lambda \in \mathbb{R}$ such that

$$
1+\left(\tan \varphi_{i}\right)^{2}=\lambda
$$

for all the non-zero angles $\varphi_{i}$. As a consequence, all the non-zero angles are necessarily equal. Investigating hence separately the cases where three, four, five and six angles are non-zero yields easily the expected result.

Let us now solve the second problem of (3.5). Let $K$ be a hexagon and let us use the notations of Fig 9. One has

$$
\min _{\Phi \in \Theta^{0}} \operatorname{Diam}(H(\Phi))=\min _{K \in \mathcal{P}_{6}} \max _{(x, y) \in K^{2}}|x-y| \geqslant \min _{K \in \mathcal{P}_{6}} \max _{i=1,2,3} A_{i} A_{i+3} .
$$


Let us now solve the problem $\min _{K \in \mathcal{P}_{6}} \max _{i=1, \ldots, 3} A_{i} A_{i+3}$. We will show that the chain of inequalities above is in fact a chain of equalities. We start by several remarks allowing to reduce the problem. Notice that the preliminary remarks of Step 1 still hold for this problem. Consider a solution denoted $K^{*}$, associated to $\Phi^{*} \in \Theta^{0}$.

- Let us assume without loss of generality that the maximum is reached by $A_{1} A_{4}$. Consider the hexagons $\hat{K}_{i}, i=1,2$, obtained by symmetrizing the quadrilaterals $A_{1} A_{2} A_{3} A_{4}$ and $A_{4} A_{5} A_{6} A_{1}$ with respect to the axis $\left(A_{1} A_{4}\right)$. Assume by contradiction that $A_{1}, A_{4}$ and $O$ are not aligned. Then, it is obvious that either the inradius of $\hat{K}_{1}$ or the one of $\hat{K}_{2}$ is strictly lower than 1. Assume that the inradius of $\hat{K}_{1}$ is strictly lower than 1 . Then, applying a well-chosen homothety to $\hat{K}_{1}$ provides a hexagon with inradius 1 having a diameter larger than the one of $K^{*}$, which is absurd. Hence, $A_{1}, A_{4}$ and $O$ are necessarily aligned and this argument can be extended to any length reaching the maximum.

- In fact, one can show that the three lengths $A_{1} A_{4}, A_{2} A_{5}$ and $A_{3} A_{6}$ are equal. Indeed, in the converse case, assume that $A_{1} A_{4}$ does not reach the maximum. We replace $A_{1}$ and $A_{4}$ by $\hat{A}_{1}$ and $\hat{A}_{4}$ that are the respective images of $A_{1}$ and $A_{4}$ by a homothety centered at the middle of $\left[A_{1} A_{4}\right]$ in such a way that $\hat{A}_{1} \hat{A}_{4}>A_{1} A_{4}$, and the maximum remains unchanged. This is a contradiction with the conclusion of Lemma 3.4.

As a result, one has necessarily $A_{1} A_{4}=A_{2} A_{5}=A_{3} A_{6}$ and moreover, the points $A_{i}, O$ and $A_{i+3}$ are aligned in this order for $i=1,2,3$. According to the considerations above, and since $O A_{i}=1 / \cos \varphi_{i}^{*}, i=1, \ldots, 6$, one has

$$
A_{i} A_{i+3}=O A_{i}+O A_{i+3}=\frac{1}{\cos \varphi_{i}^{*}}+\frac{1}{\cos \varphi_{i+3}^{*}} .
$$

Therefore, we infer that

$$
\min _{\Phi \in \Theta^{0}} \operatorname{Diam}(H(\Phi)) \geqslant \min _{K \in \mathcal{P}_{6}} \max _{i=1,2,3} A_{i} A_{i+3}=\max _{i=1,2,3}\left(\frac{1}{\cos \varphi_{i}^{*}}+\frac{1}{\cos \varphi_{i+3}^{*}}\right) .
$$

Moreover, one has

$$
\max _{i=1,2,3} \frac{1}{\cos \varphi_{i}^{*}}+\frac{1}{\cos \varphi_{i+3}^{*}} \geqslant \frac{1}{3} \sum_{i=1}^{6} \frac{1}{\cos \varphi_{i}^{*}} \geqslant \frac{1}{3} \min _{K \in \mathcal{P}_{6}} \sum_{i=1}^{6} \frac{1}{\cos \varphi_{i}} .
$$

For this last problem, let $\tilde{\Phi}$ be a solution. Notice that one has necessarily $\tilde{\varphi}_{i}<\pi / 2$. Let us assume that $\tilde{\varphi}_{i}$ is positive. Hence, it follows from the Karush-Kuhn-Tucker theorem the existence of a Lagrange multiplier $\lambda \in \mathbb{R}$ such that

$$
-\frac{\sin \varphi_{i}}{\cos ^{2} \varphi_{i}}=\lambda
$$

and therefore, all the non-zero angles must be equal. For $N=3,4,5,6$, assume that there are $6-N$ zero angles and $N$ nonzero angles (therefore equal to $\pi / N$ according to the equality constraint). One shows easily that

$$
\sum_{i=1}^{6} \frac{1}{\cos \tilde{\varphi}_{i}}=\frac{N}{\cos (\pi / N)}+(N-6) \geqslant \frac{6}{\cos (\pi / 6)}=\frac{12}{\sqrt{3}} .
$$


This proves that the only solution of the problem $\min _{K \in \mathcal{P}_{6}} \sum_{i=1}^{6} \frac{1}{\cos ^{2} \varphi_{i}}$ is $\tilde{\Phi}=$ $\frac{\pi}{6}(1,1,1,1,1,1)$. We infer from this reasoning that

$$
\min _{\Phi \in \Theta^{0}} \operatorname{Diam}(H(\Phi)) \geqslant \frac{4}{\sqrt{3}} .
$$

We conclude by noting that this inequality is an equality as soon as $\Phi=\tilde{\Phi}$ (in other words, whenever $K^{*}$ is a regular hexagon with inradius 1 ).

Step 3. Solving Problem (3.6). Assume that $K^{*}$ is the hexagon plotted on Fig. 9. The diameter can be realized in three ways: (i) on a side, (ii) on a diagonal of the kind $A_{1} A_{4}$, or (iii) on a diagonal of the kind $A_{1} A_{3}$. In what follows, we will first consider separately each of these three cases and combine them in a second time to get the expected result. In the sequel, we will denote by $\Phi^{*}=\left(\varphi_{1}^{*}, \ldots, \varphi_{6}^{*}\right)$ a solution of (3.6) associated to a hexagon $K^{*}$.

Case (i): the diameter is realized by a side

Assume without loss of generality that the diameter of $K^{*}$ is given by $A_{1} A_{2}$ (this is always possible by re-indexing the vertices). For $\Phi \in \Theta_{A}$, denote by $D_{1,2}(\Phi)$ the length $A_{1} A_{2}$ in the hexagon $H(\Phi)$. One has $D_{1,2}(\Phi)=\tan \left(\varphi_{1}\right)+\tan \left(\varphi_{2}\right)$, and we are therefore led to solve the optimization problem

$$
\max _{\Phi \in \Theta} \tan \left(\varphi_{1}\right)+\tan \left(\varphi_{2}\right) .
$$

It is notable that for the hexagon $K^{*}$, one has necessarily $0<\varphi_{i}<\pi / 2$. Indeed, the left inequality is a direct consequence of the conclusion of Lemma 3.1 for Problem (3.4), and the right one comes from the area constraint. According to the KarushKuhn-Tucker theorem, there exists $(\lambda, \mu) \in \mathbb{R}^{2}$ such that $1+\tan ^{2}\left(\varphi_{i}^{*}\right)=\lambda(1+$ $\left.\tan ^{2}\left(\varphi_{i}^{*}\right)\right)+\mu$ for $i=1,2$, and $0=\lambda\left(1+\tan ^{2}\left(\varphi_{i}^{*}\right)\right)+\mu$ for $i=3,4,5,6$.

The two first equations yield $(\lambda, \mu) \neq(0,0)$ and we easily infer that

$$
\varphi_{1}^{*}=\varphi_{2}^{*} \quad \text { and } \quad \varphi_{3}^{*}=\varphi_{4}^{*}=\varphi_{5}^{*}=\varphi_{6}^{*} .
$$

Denoting by $\varphi$ the angle $\varphi_{1}^{*}$ and by $\psi$ the angle $\varphi_{3}^{*}$, it follows from the equality constraint on the $\varphi_{i}$ 's and from the area constraint that

$$
\psi=\frac{\pi}{4}-\frac{\varphi}{2} \quad \text { and } \quad 2 \tan (\varphi)+4 \tan (\psi)=A
$$

Let $t=\tan (\varphi / 2)$. Since

$$
\tan (\psi)=\tan \left(\frac{\pi}{4}-\frac{\varphi}{2}\right)=\frac{1-\tan (\varphi / 2)}{1+\tan (\varphi / 2)}=\frac{1-t}{1+t},
$$

the second equation rewrites $4\left(\frac{t}{1-t^{2}}+\frac{1-t}{1+t}\right)=A$, and hence

$$
t^{2}\left(1+\frac{A}{4}\right)-t+1-\frac{A}{4}=0 .
$$

Since $A \geqslant 2 \sqrt{3}$, this equation has two real roots, and the largest one is

$$
t=\frac{1+\sqrt{\frac{A^{2}}{4}-3}}{2\left(1+\frac{A}{4}\right)} .
$$


We then get

$$
\max _{\Phi \in \Theta_{A}} D_{1,2}(\Phi)=D_{1,2}\left(\Phi^{*}\right)=\frac{4 t}{1-t^{2}}
$$

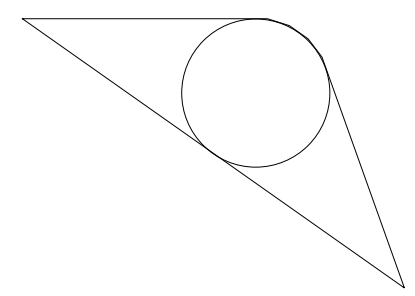

FIG. 10. Hexagon maximizing $D_{1,2}(\Phi)$ for $A=4 \sqrt{3}$

Case (ii): the diameter is realized by $A_{1} A_{4}$

Since $O A_{1}=1 / \cos \varphi_{1}, O A_{4}=1 / \cos \varphi_{4}$ and $\widehat{A_{1} O A_{4}}=\varphi_{1}+2 \varphi_{2}+2 \varphi_{3}+\varphi_{4}$, one has $A_{1} A_{4}^{2}=D_{1,4}(\Phi)$ where

$$
D_{1,4}(\Phi)=\frac{1}{\cos ^{2} \varphi_{1}}+\frac{1}{\cos ^{2} \varphi_{4}}-\frac{2 \cos \left(\varphi_{1}+2 \varphi_{2}+2 \varphi_{3}+\varphi_{4}\right)}{\cos \varphi_{1} \cos \varphi_{4}},
$$

by using the Al-Kashi formula in the triangle $A_{1} O A_{4}$. Notice that for all $\Phi \in \Theta$, one has

$$
D_{1,4}(\Phi) \leqslant \frac{1}{\cos ^{2} \varphi_{1}}+\frac{1}{\cos ^{2} \varphi_{4}}+\frac{2}{\cos \varphi_{1} \cos \varphi_{4}}=\left(\frac{1}{\cos \varphi_{1}}+\frac{1}{\cos \varphi_{4}}\right)^{2}=G\left(\varphi_{1}, \varphi_{4}\right)^{2},
$$

where $G(x, y)=\frac{1}{\cos (x)}+\frac{1}{\cos (y)}$ for all $x, y \in[0, \pi / 2]^{2}$.

To solve the problem of maximizing $D_{1,4}$ over $\Theta_{A}$, we will maximize the mapping $\Phi \mapsto G\left(\varphi_{1}, \varphi_{4}\right)^{2}$ over $\Theta_{A}$ and use (3.7) to prove that both the optimal values and the maximizers of the aforementioned problems coincide. Hence, we investigate the optimization problem

$$
\max _{\Phi \in \Theta_{A}} G\left(\varphi_{1}, \varphi_{4}\right) .
$$

With a slight abuse of notation, we denote by $\Phi^{*}$ a solution to this problem. Reasoning similarly as for the case (i), we first notice that one has necessarily $\varphi_{i}^{*} \in\left(0, \frac{\pi}{2}\right)$. Applying the Karush-Kuhn-Tucker theorem, we infer the existence of $(\lambda, \mu) \in \mathbb{R}^{2}$ such that

$$
\frac{\sin \left(\varphi_{i}^{*}\right)}{\cos ^{2}\left(\varphi_{i}^{*}\right)}=\lambda\left(1+\tan ^{2}\left(\varphi_{i}^{*}\right)\right)+\mu
$$

for $i=1,4$, whereas $0=\lambda\left(1+\tan ^{2}\left(\varphi_{i}^{*}\right)\right)+\mu$ for $i=2,3,5,6$. By exploiting these equalities, we get successively that $\varphi_{2}^{*}=\varphi_{3}^{*}=\varphi_{5}^{*}=\varphi_{6}^{*}, \mu=-\lambda\left(1+\tan ^{2}\left(\varphi_{2}\right)\right)$, and that $\varphi_{1}^{*}$ and $\varphi_{4}^{*}$ solve the equation

$$
\frac{\sin \theta}{\cos ^{2} \theta}=\lambda\left(\tan ^{2} \theta-\tan ^{2}\left(\varphi_{2}^{*}\right)\right)
$$


Notice that $\varphi_{1}^{*} \neq \varphi_{2}^{*}$. Indeed, in the converse case, one has $\varphi_{1}^{*}=0=\varphi_{2}^{*}=\varphi_{3}^{*}=\varphi_{5}^{*}=$ $\varphi_{6}^{*}$ and then $\varphi_{4}^{*}=\pi$, which is absurd. Similarly, one has $\varphi_{4}^{*} \neq \varphi_{2}^{*}$. Equation (3.8) hence rewrites

$$
\frac{\sin \theta}{\cos ^{2} \theta\left(\tan ^{2} \theta-\tan ^{2}\left(\varphi_{2}\right)\right)}=\lambda
$$

We claim that the function $h$ defined by

$$
h: \theta \in\left[0, \varphi_{2}^{*}\right) \cup\left(\varphi_{2}^{*}, \frac{\pi}{2}\right) \mapsto \frac{\sin \theta}{\cos ^{2} \theta\left(\tan ^{2} \theta-\tan ^{2}\left(\varphi_{2}^{*}\right)\right)} .
$$

is one-to-one ${ }^{3}$. As a result, one has $\varphi_{1}^{*}=\varphi_{4}^{*}$, and we infer that

$$
\varphi_{1}^{*}=\varphi_{4}^{*}=2 \arctan \left(\frac{1+\sqrt{\frac{A^{2}}{4}-3}}{2\left(1+\frac{A}{4}\right)}\right) \quad \text { and } \quad \varphi_{2}^{*}=\varphi_{3}^{*}=\varphi_{5}^{*}=\varphi_{6}^{*}=\frac{\pi}{4}-\frac{\varphi_{1}^{*}}{2} .
$$

Noticing that $\varphi_{1}^{*}+2 \varphi_{2}^{*}+2 \varphi_{3}^{*}+\varphi_{4}^{*}=\pi$ and according to the previous considerations, it follows that

$$
\max _{\Phi \in \Theta_{A}} D_{1,4}(\Phi)=D_{1,4}\left(\Phi^{*}\right)=\left(\frac{1}{\cos \varphi_{1}^{*}}+\frac{1}{\cos \varphi_{4}^{*}}\right)^{2}=G^{2}\left(\varphi_{1}^{*}, \varphi_{4}^{*}\right)=\max _{\Phi \in \Theta_{A}} G^{2}\left(\varphi_{1}, \varphi_{4}\right) .
$$

Moreover, the maximal value of $A_{1} A_{4}$ is $2 / \cos \varphi_{1}^{*}$.

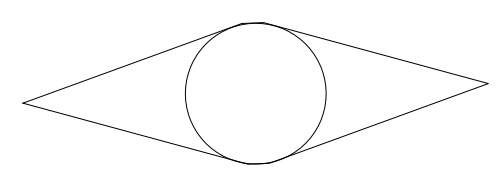

FIG. 11. Hexagon maximizing $A_{1} A_{4}$ for $A=4 \sqrt{3}$

Case (iii): the diameter is realized by $A_{1} A_{3}$

Using similar computations as for $A_{1} A_{4}$, we get

$$
A_{1} A_{3}^{2}=D_{1,3}(\varphi), \quad \text { with } \quad D_{1,3}(\varphi)=\frac{1}{\cos ^{2} \varphi_{1}}+\frac{1}{\cos ^{2} \varphi_{3}}-\frac{2 \cos \left(\varphi_{1}+2 \varphi_{2}+\varphi_{3}\right)}{\cos \varphi_{1} \cos \varphi_{3}} .
$$

Following exactly the same lines as for the case (ii), and using the same notations, one shows successively that $\forall \Phi \in \Theta_{A}, D_{1,3}(\Phi) \leqslant G^{2}\left(\varphi_{1}, \varphi_{3}\right)$ and

$$
D_{1,4}\left(\Phi^{*}\right)=\max _{\Phi \in \Theta_{A}} D_{1,4}(\Phi)=\max _{\Phi \in \Theta_{A}} G^{2}\left(\varphi_{1}, \varphi_{4}\right)=\max _{\Phi \in \Theta_{A}} G^{2}\left(\varphi_{1}, \varphi_{3}\right)
$$

\footnotetext{
${ }^{3}$ Indeed, since $h$ is negative on $\left[0, \varphi_{2}\right)$ and positive on $\left(\varphi_{2}^{*}, \pi / 2\right)$, we can deal separately with the intervals $\left[0, \varphi_{2}^{*}\right)$ and $\left(\varphi_{2}^{*}, \pi / 2\right)$. On $\left[0, \varphi_{2}^{*}\right)$, one has $h(\theta)=\sin \theta \frac{1+\tan ^{2} \theta}{\tan ^{2} \theta-\tan ^{2}\left(\varphi_{2}^{*}\right)}$. It follows that $h$ is the product of the positive increasing sine function by $\theta \mapsto \frac{1+\tan ^{2} \theta}{\tan ^{2} \theta-\tan ^{2}\left(\varphi_{2}^{*}\right)}$, which is negative decreasing. The conclusion follows.

On $\left(\varphi_{2}^{*}, \pi / 2\right)$, one has $h(\theta)=\frac{1}{\sin \theta}\left(1-\frac{\tan ^{2}\left(\varphi_{2}^{*}\right)}{\tan ^{2} \theta}\right)^{-1}$, and therefore, $h$ is the product of two positive decreasing functions, whence the result.
} 
As a consequence, there holds

$$
\max _{\Phi \in \Theta_{A}} D_{1,3}(\Phi) \leqslant \max _{\Phi \in \Theta_{A}} D_{1,4}(\Phi)
$$

with equality if, and only if there exists $\Phi^{*} \in \Theta$ such that $\pi=\varphi_{1}^{*}+2 \varphi_{2}^{*}+\varphi_{3}^{*}$. Because of the first equality constraint on the angles $\varphi_{i}$, it follows that $\varphi_{2}^{*}=\varphi_{4}^{*}+\varphi_{5}^{*}+\varphi_{6}^{*}$. Now, writing the optimality conditions for the problem of maximizing $D_{1,3}$ over $\Theta_{A}$ as for the case (ii), we infer that $\varphi_{2}^{*}=\varphi_{4}^{*}=\varphi_{5}^{*}=\varphi_{6}^{*}$. Thus, these angles are necessarily equal to 0 , which contradicts Lemma 3.1. This shows that the case (iii) cannot arise.

Comparison between the three cases

According to the previous analysis, one has $A_{1} A_{4}>A_{1} A_{3}$ for any optimal set $K^{*}$. Notice moreover that $\max _{\Phi \in \Theta_{A}} A_{1} A_{2}=2 \tan \left(\Phi^{*}\right)$ and $\max _{\Phi \in \Theta_{A}} A_{1} A_{4}=\frac{2}{\cos \left(\Phi^{*}\right)}$ with $\Phi^{*}=2 \arctan \left(\frac{1+\sqrt{\frac{A^{2}}{4}-3}}{2\left(1+\frac{A}{4}\right)}\right)$. We then infer that the solution of Problem (3.6) corresponds to the case (ii).

Therefore, the optimization problem has a unique solution (whenever $A \geqslant 2 \sqrt{3} r_{0}^{2}$ ) given by the hexagon with inner radius $r_{0}$, which is tangent at every side to its inner circle, and such that the semi circle center-angles are given by

$$
\varphi_{1}^{*}=\varphi_{4}^{*}=2 \arctan \left(\frac{1+\sqrt{\frac{A^{2}}{4 r_{0}^{4}}-3}}{2\left(1+\frac{A}{4 r_{0}^{2}}\right)}\right) \quad \text { and } \quad \varphi_{2}^{*}=\varphi_{3}^{*}=\varphi_{5}^{*}=\varphi_{6}^{*}=\frac{\pi}{4}-\frac{\varphi_{1}^{*}}{2} .
$$

4. Proof of Theorem 2.9. Before solving Problem (1.3), we first investigate the following auxiliary problem:

$$
\max \{d(K),|K|=A, r(K)=r, \operatorname{Diam}(K)=D\},
$$

where $(A, D, r)$ denote the triple of positive numbers.

To help the forthcoming analysis and since several cases must be distinguished, let us plot on Fig. 12 some elements of the Blaschke-Santaló diagram for the diameter and inradius, the area being fixed.
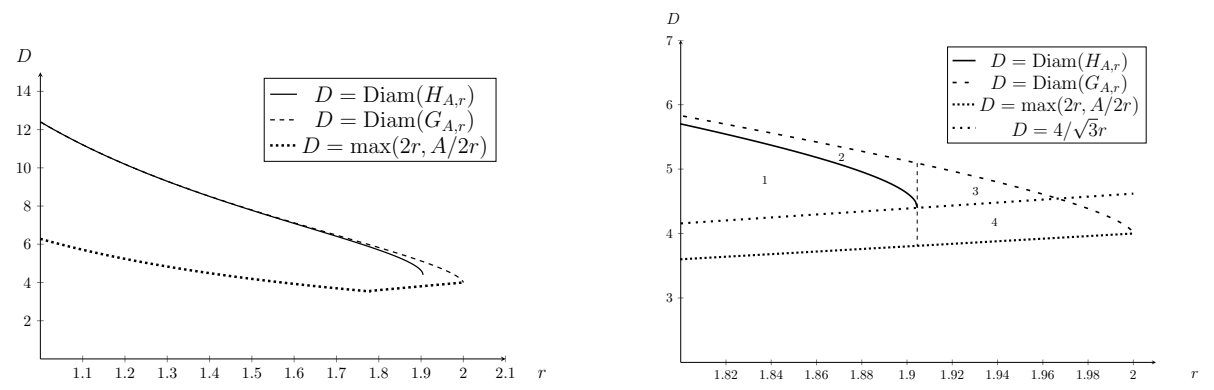

FIG. 12. Left: Blaschke-Santaló Diagram for $(r(K), \operatorname{Diam}(K))$ under the condition $A=|K|=$ $4 \pi$. Right: zoom on the right part of the diagram.

Remark 4.1. Let us comment on the construction of Fig. 12. The green boundary consists of two parts. The first one is obtained by using that for every convex set $K$, one has

$$
|K|<2 \operatorname{Diam}(K) r(K),
$$


with equality if, and only if $\operatorname{int}(K)=\emptyset$ (see [6]), and the (straight) right one is obtained by using that $\operatorname{Diam}(K) \geqslant 2 r(K)$ with equality if, and only if $K$ is a ball. The red boundary is determined by using the second item of Theorem 2.7. Finally, the blue boundary is obtained by using (2.5) in Remark 2.5.

First, notice that, according to the so-called isodiametric inequality, one has $r \leqslant \operatorname{Diam}(K) / 2 \leqslant \sqrt{A / \pi}$ and $\operatorname{Diam}(K) \leqslant \operatorname{Diam}\left(G_{A, r}\right)$ for every convex body $K$ having as inradius $r$ and area $A$, where the 2-cap body $G_{A, r}$ has been introduced in Definition 2.4.

The main ingredient of the proof of Theorem 2.9 is the following lemma about the maximization of the density functional $d(\cdot)$, whose proof is postponed at the end of this section for the sake of clarity.

Lemma 4.2. Let $A>0$ and $D>0$.

1. Let $r \in(0, \sqrt{A / 2 \sqrt{3}}]$ and $D=\operatorname{Diam}\left(H_{A, r}\right)$. One has

$$
\max \{d(K), r(K)=r, \operatorname{Diam}(K) \leqslant D,|K|=A\}=1 .
$$

2. Let $r \in(0, \sqrt{A / 2 \sqrt{3}}]$ and $D>\operatorname{Diam}\left(H_{A, r}\right)$ or $(r, D) \in\{(r, D) \mid r>$ $\sqrt{A / 2 \sqrt{3}}$ and $\left.D \in\left[4 / \sqrt{3} r, \operatorname{Diam}\left(G_{A, r}\right)\right]\right\}$. Then, one has

$$
\max \{d(K), r(K)=r, \operatorname{Diam}(K)=D,|K|=A\}=\frac{A}{\left|H^{D, r}\right|} .
$$

3. Let $(r, D) \in\left\{(r, D) \mid r>\sqrt{A / 2 \sqrt{3}}\right.$ and $\left.D \leqslant \min \left\{4 / \sqrt{3} r, \operatorname{Diam}\left(G_{A, r}\right)\right\}\right\}$. Then, one has

$$
\max \{d(K), r(K)=r, \operatorname{Diam}(K)=D,|K|=A\}=\frac{A}{\left|H_{r}^{*}\right|} .
$$

Let us come back to the solution of Problem (1.3).

Let us distinguish between several cases, depending on the possible values of $r(K)$ and $\operatorname{Diam}(K)$. For that purpose, let us notice that

$$
\sup _{K \in \mathcal{A}_{r_{0}, A}} J_{t}(K)=\max _{1 \leqslant i \leqslant 4} \sup _{K \in \mathcal{A}_{r_{0}, A}^{i}} J_{t}(K)
$$

with the following partition of $\mathcal{A}_{r_{0}, A}$ :

$$
\begin{aligned}
& \left.\mathcal{A}_{r_{0}, A}^{1}=\left\{K \in \mathcal{A}_{r_{0}, A} \mid r(K)=r, r_{0} \leqslant r \leqslant \sqrt{A /(2 \sqrt{3})} \text { and } \operatorname{Diam}(K) \leqslant D_{H_{A, r}}\right)\right\} \\
& \mathcal{A}_{r_{0}, A}^{2}=\left\{K \in \mathcal { A } _ { r _ { 0 } , A } | r ( K ) = r , r _ { 0 } \leqslant r \leqslant \sqrt { A / ( 2 \sqrt { 3 } ) } \text { and } \operatorname { D i a m } ( K ) \in \left(D_{\left.\left.H_{A, r}, D_{G_{A, r}}\right]\right\}}\right.\right. \\
& \mathcal{A}_{r_{0}, A}^{3}=\left\{K \in \mathcal{A}_{r_{0}, A} \mid r(K)=r, r>\sqrt{A /(2 \sqrt{3})} \text { and } \operatorname{Diam}(K) \in\left(4 / \sqrt{3} r, D_{G_{A, r}}\right)\right\} \\
& \mathcal{A}_{r_{0}, A}^{4}=\left\{K \in \mathcal{A}_{r_{0}, A} \mid r(K)=r, r>\sqrt{A /(2 \sqrt{3})} \text { and } \operatorname{Diam}(K) \leqslant 4 / \sqrt{3} r\right\} .
\end{aligned}
$$

where we introduce the notations $D_{H_{A, r}}=\operatorname{Diam}\left(H_{A, r}\right)$ and $D_{G_{A, r}}=\operatorname{Diam}\left(G_{A, r}\right)$, for the sake of readability. $\mathcal{A}_{r_{0}, A}^{i}$ corresponds to the zone $i$ in Figure 12.

Let us investigate each problem separately. 
Solution of Problem $\sup _{K \in \mathcal{A}_{r_{0}, A}^{1}} J_{t}(K)$. Let $r \in\left[r_{0}, \sqrt{A /(2 \sqrt{3})}\right]$ and $K \in \mathcal{A}_{r_{0}, A}^{1}$ such that $r(K)=r$. According to Lemma 4.2, one has

$$
J_{t}(K) \leqslant t+(1-t) \frac{\sqrt{\pi} D_{H_{A, r}}}{2 \sqrt{A}}=t+(1-t) \frac{\sqrt{\pi}}{2 \sqrt{A}}\left(\frac{1}{3 r}\left(2 A+\sqrt{A^{2}-12 r^{4}}\right)\right)
$$

with equality if $K=H_{A, r}$. Moreover, the mapping $r \mapsto \frac{1}{3 r}\left(2 A+\sqrt{A^{2}-12 r^{4}}\right)$ is decreasing on $(0,+\infty)$. As a consequence, we infer that

$$
\max _{K \in \mathcal{A}_{r_{0}, A}^{1}} J_{t}(K)=J_{t}\left(H_{A, r}\right)=t+(1-t) \frac{\sqrt{\pi}}{2 \sqrt{A}}\left(\frac{1}{3 r_{0}}\left(2 A+\sqrt{A^{2}-12 r_{0}^{4}}\right)\right),
$$

and the maximum is reached by the $p$-hexagon $H_{A, r_{0}}$.

Solution of Problem $\sup _{K \in \mathcal{A}_{r_{0}, A}^{2} \cup \mathcal{A}_{r_{0}, A}^{3}} J_{t}(K)$. Let $K \in \mathcal{A}_{r_{0}, A}^{2}$ and $r=r(K)$ be such that $r \in\left[r_{0}, \sqrt{A /(2 \sqrt{3})}\right]$. According to Lemma 4.2, one has

$$
J_{t}(K) \leqslant t \frac{A}{\left|H^{D, r}\right|}+(1-t) \frac{\sqrt{\pi} D}{2 \sqrt{A}}
$$

Let us first maximize the function in the right-hand side, by solving the problem

$$
\max _{(D, r) \in \mathcal{Z}} \psi_{t, A}(r, D) \quad \text { where } \quad \psi_{t, A}(r, D)=t \frac{A}{\left|H^{D, r}\right|}+(1-t) \frac{\sqrt{\pi} D}{2 \sqrt{A}},
$$

with

$$
\begin{array}{r}
\mathcal{Z}=\left\{(r, D) \mid r_{0} \leqslant r \leqslant \sqrt{A /(2 \sqrt{3})} \text { and } D \in\left(D_{H_{A, r}}, D_{G_{A, r}}\right)\right. \\
\quad \text { or } r>\sqrt{A /(2 \sqrt{3})} \text { and } D \geqslant 4 / \sqrt{3} r\} .
\end{array}
$$

This corresponds to deal with the zone 2 and 3 of Fig. 12. First, note that

$$
\frac{d \psi_{t, A}}{d r}(r, D)=\frac{-t A\left(2 D \sqrt{D^{2}-4 r^{2}}-D^{2}+8 r^{2}\right)}{\sqrt{D^{2}-4 r^{2}}\left(2 D r-r \sqrt{D^{2}-4 r^{2}}\right)^{2}} .
$$

Moreover, if $D^{2} \leqslant 8 r^{2}$, we conclude directly that $2 D \sqrt{D^{2}-4 r^{2}}-D^{2}+8 r^{2}$ is positive. In the converse case, the sign of $2 D \sqrt{D^{2}-4 r^{2}}-D^{2}+8 r^{2}$ is also the sign of $4 D^{2}\left(D^{2}-\right.$ $\left.4 r^{2}\right)-\left(D^{2}-8 r^{2}\right)^{2}$, namely $3 D^{4}-64 r^{4}$. Notice that, in that zone, one has $D \geqslant$ $4 r / \sqrt{3} \geqslant r \sqrt{8 / \sqrt{3}}$, which means precisely that $3 D^{4}-64 r^{4}>0$. In all cases, we then have $2 D \sqrt{D^{2}-4 r^{2}}-D^{2}+8 r^{2}>0$, and we infer that $\frac{d \psi_{t, A}}{d r}(r, D)<0$. It follows that either $D=D_{H_{A, r}}$ or $r=r_{0}$. The case $D=D_{H_{A, r}}$ has been investigated when solving Problem $\sup _{K \in \mathcal{A}_{r_{0}, A}^{1}} J_{t}(K)$ above. As a consequence, one has necessarily $r=r_{0}$ at the maximum.

It then remains to investigate the variations of the criterion with respect to the parameter $D$, at $r=r_{0}$. One has

$$
\frac{d^{2} \psi_{t, A}}{d D^{2}}\left(r_{0}, D\right)=-\frac{2 A t\left(\sqrt{D^{2}-4 r_{0}^{2}}\left(14 r_{0}^{2}-5 D^{2}\right)+4 D\left(D^{2}-3 r_{0}^{2}\right)\right)}{r_{0}\left(D^{2}-4 r_{0}^{2}\right)^{3 / 2}\left(2 D-\sqrt{D^{2}-4 r_{0}^{2}}\right)^{3}} .
$$


Note that $\sqrt{D^{2}-4 r_{0}^{2}}\left(14 r_{0}^{2}-5 D^{2}\right)+4 D\left(D^{2}-3 r_{0}^{2}\right)=r_{0}^{3}\left(\sqrt{X^{2}-4}\left(14-5 X^{2}\right)+4 X\left(X^{2}-3\right)\right)$ with $X=D / r_{0}$. Recall that the function $X \mapsto \sqrt{X^{2}-4}\left(14-5 X^{2}\right)+4 X\left(X^{2}-3\right)$ has a unique zero $X_{0}$ on $[4 / \sqrt{3},+\infty)$. Moreover, a tedious but easy analysis yields that $\sqrt{X^{2}-4}\left(14-5 X^{2}\right)+4 X\left(X^{2}-3\right) \geqslant 0$ on $\left[4 / \sqrt{3}, X_{0}\right]$ and $\sqrt{X^{2}-4}\left(14-5 X^{2}\right)+$ $4 X\left(X^{2}-3\right)<0$ elsewhere.

It follows that the mapping $D \mapsto \frac{d}{d D} \psi_{t, A}\left(r_{0}, \cdot\right)$ is decreasing on $\left[4 \sqrt{3} r_{0}, X_{0} r_{0}\right]$ and increasing on $\left[X_{0} r_{0},+\infty\right)$. Its minimal value is

$$
\frac{d \psi_{t, A}}{d D}\left(r_{0}, X_{0} r_{0}\right)=\frac{-A \gamma_{0}}{r_{0}^{3}} t+(1-t) \frac{\sqrt{\pi}}{2 \sqrt{A}}
$$

where $\gamma_{0}$ is defined by (2.9).

The minimal value of $\frac{d \psi_{t, A}}{d D}\left(r_{0}, \cdot\right)$ is then non-negative whenever $t \in\left[0, t_{A, r_{0}}\right]$ and negative whenever $t \in\left(t_{A, r_{0}}, 1\right]$, where $t_{A, r_{0}}$ is given by (2.8). If $t \in\left[0, t_{A, r_{0}}\right]$, we infer from the above analysis that $D \mapsto \psi_{t, A}\left(r_{0}, D\right)$ is increasing on $\left(D_{H_{A, r}}, D_{G_{A, r}}\right)$ and the maximum is achieved at $D=D_{G_{A, r}}$. that

If $t \in\left(t_{A, r_{0}}, 1\right]$, the minimal value of $\frac{d \psi_{t, A}}{d D} \psi_{t, A}\left(r_{0}, \cdot\right)$ is negative. Notice moreover

$$
\frac{d \psi_{t, A}}{d D}\left(r_{0}, 4 r_{0} / \sqrt{3}\right)=\lim _{D \rightarrow+\infty} \frac{d \psi_{t, A}}{d D}\left(r_{0}, D\right)=\frac{(1-t) \sqrt{\pi}}{2 \sqrt{A}} .
$$

Combining these informations about $\frac{d \psi_{t, A}}{d D}$ yields the existence ${ }^{4}$ of $z_{t, r_{0}, A}^{1} \in\left[4 / \sqrt{3}, X_{0}\right)$ and $z_{t, r_{0}, A}^{2} \in\left[X_{0},+\infty\right)$ such that the mapping $D \mapsto \psi_{t, A}\left(r_{0}, \cdot\right)$ is increasing on $\left(4 / \sqrt{3} r_{0}, z_{t, r_{0}, A}^{1} r_{0}\right)$, decreasing on $\left(z_{t, r_{0}, A}^{1}, z_{t, r_{0}, A}^{2}\right)$ and increasing on $\left(z_{t, r_{0}, A}^{2},+\infty\right)$.

Now, using that $\operatorname{Diam}\left(H_{A, r_{0}}\right)=\frac{1}{3 r_{0}}\left(2 A+\sqrt{A^{2}-12 r_{0}^{4}}\right)$ and that the mapping $[2 \sqrt{3},+\infty) \ni A \mapsto \frac{1}{3 r_{0}}\left(2 A+\sqrt{A^{2}-12 r_{0}^{4}}\right)$ is increasing, we claim that

$$
\frac{A}{r_{0}^{2}} \geqslant 2 X_{0}-\sqrt{X_{0}^{2}-4} \Longleftrightarrow D_{H_{A, r_{0}}} \geqslant X_{0} r_{0}
$$

Since $\psi_{t, A}\left(r_{0}, \cdot\right)$ decreases on $\left[X_{0} r_{0}, z_{t, r_{0}, A}^{2}\right]$ and increases on $\left(z_{t, r_{0}, A}^{2},+\infty\right)$, we infer that, under the smallness condition (2.10) on $r_{0}$, one has successively

$$
\begin{aligned}
\max _{(D, r) \in \mathcal{Z}} \psi_{t, A}(r, D) & =\max _{D \in\left(D_{H_{A, r_{0}}}, D_{G_{A}, r_{0}}\right)} \psi_{t, A}\left(r_{0}, D\right) \\
& =\max \left\{\psi_{t, A}\left(r_{0}, D_{H_{A, r_{0}}}\right), \psi_{t, A}\left(r_{0}, D_{G_{A, r_{0}}}\right)\right\}
\end{aligned}
$$

To solve the problem arising in the right-hand side, let us introduce

$$
\Delta_{r_{0}, A}(t)=\psi_{t, A}\left(r_{0}, D_{G_{A, r_{0}}}\right)-\psi_{t, A}\left(r_{0}, D_{H_{A, r_{0}}}\right) .
$$

One computes

$$
\Delta_{r_{0}, A}(0)=\frac{\sqrt{\pi}}{2 \sqrt{A}}\left(D_{G_{A, r_{0}}}-D_{H_{A, r_{0}}}\right), \quad \Delta_{r_{0}, A}(1)=A\left(\frac{1}{\left|D_{G_{A, r_{0}}}\right|}-\frac{1}{\left|D_{H_{A, r_{0}}}\right|}\right) .
$$

\footnotetext{
${ }^{4}$ Moreover, $z_{t, r_{0}, A}^{1}$ and $z_{t, r_{0}, A}^{2}$ are the two solutions of the equation $d \psi_{t, A} / d D\left(r_{0}, r_{0} z\right)=0$ with unknown $z$ on $[4 / \sqrt{3},+\infty)$

$$
\frac{2 \sqrt{z^{2}-4}-z}{\sqrt{z^{2}-4}\left(2 z-\sqrt{z^{2}-4}\right)^{2}}=\frac{(1-t) \sqrt{\pi} r_{0}^{3}}{2 t A^{3 / 2}}
$$
}


Let $M_{t, r_{0}, A}=\max \left\{\psi_{t, A}\left(r_{0}, D_{H_{A, r_{0}}}\right), \psi_{t, A}\left(r_{0}, D_{G_{A, r_{0}}}\right)\right\}$. Hence, since $\Delta_{r_{0}, A}$ is affine, $\Delta_{r_{0}, A}(0)>0, \Delta_{r_{0}, A}(1)<0$, we infer the existence of $t_{A, r_{0}}^{*} \in[0,1]$ such that on $\left[0, t_{A, r_{0}}^{*}\right], M_{t, r_{0}, A}=\psi_{t, A}\left(r_{0}, D_{G_{A, r_{0}}}\right)$; on $\left(t_{A, r_{0}}^{*}, 1\right], M_{t, r_{0}, A}=\psi_{t, A}\left(r_{0}, D_{H_{A, r_{0}}}\right)$. Notice that, by construction, one has $\Delta_{r_{0}, A}\left(t_{A, r_{0}}^{*}\right)=0$ leading to the expression (2.11) of $t_{A, r_{0}}^{*}$ and one has necessarily $t_{A, r_{0}}^{*} \geqslant t_{A, r_{0}}$ according to the analysis of the case where $t \in\left[0, t_{A, r_{0}}\right]$.

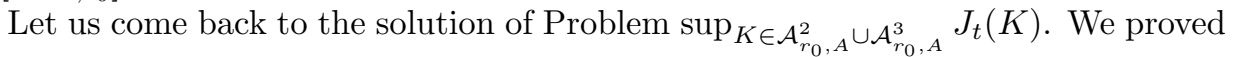
that, under the smallness assumption (2.10) on $r_{0}, G_{A, r_{0}}$ and $H_{A, r_{0}}$ are the only possible solutions of Problem $\max _{(D, r) \in \mathcal{Z}} \psi_{t, A}(r, D)$. Noting that (4.2) is an equality whenever $K$ is either equal to $G_{A, r_{0}}$, or $H_{A, r_{0}}$, we infer to the end that

$$
\max _{K \in \mathcal{A}_{r_{0}, A}^{2} \cup \mathcal{A}_{r_{0}, A}^{3}} J_{t}(K)= \begin{cases}J_{t}\left(G_{A, r_{0}}\right) & \text { if } t \in\left[0, t_{A, r_{0}}^{*}\right] \\ J_{t}\left(H_{A, r_{0}}\right) & \text { if } t \in\left(t_{A, r_{0}}^{*}, 1\right] .\end{cases}
$$

Estimate of $\sup _{K \in \mathcal{A}_{r_{0}, A}^{4}} J_{t}(K)$. According to Lemma 4.2, one has

$$
J_{t}(K) \leqslant t \frac{A}{\left|H_{r}^{*}\right|}+(1-t) \frac{\sqrt{\pi} D}{2 \sqrt{A}}=t \frac{A \sqrt{3}}{2 r^{2}}+(1-t) \frac{\sqrt{\pi} D}{2 \sqrt{A}} .
$$

Since $D \mapsto t \frac{A \sqrt{3}}{2 r^{2}}+(1-t) \frac{\sqrt{\pi} D}{2 \sqrt{A}}$ is increasing, we infer that the solutions of the problem

$$
\max _{(r, D) \in \mathcal{Z}} t \frac{A \sqrt{3}}{2 r^{2}}+(1-t) \frac{\sqrt{\pi} D}{2 \sqrt{A}}
$$

with $\hat{\mathcal{Z}}=\{(r, D) \mid \sqrt{A /(2 \sqrt{3})} \leqslant r \leqslant \sqrt{A / \pi}$ and $2 r \leqslant D \leqslant 4 / \sqrt{3} r\}$, satisfy necessarily $D=4 r / \sqrt{3}$. According to Lemma 4.2 , we deduce successively that

$$
\begin{aligned}
\max _{K \in \mathcal{A}_{r_{0}, A}^{4}} J_{t}(K) & =\max _{(r, D) \in \hat{\mathcal{Z}}} t \frac{A \sqrt{3}}{2 r^{2}}+(1-t) \frac{\sqrt{\pi} D}{2 \sqrt{A}} \\
& \leqslant \max _{K \in \mathcal{A}_{r_{0}, A}^{2} \cup \mathcal{A}_{r_{0}, A}^{3}} J_{t}(K) .
\end{aligned}
$$

Moreover, we have proved that every solution of the last problem in the right-hand side must satisfy $r(K)=r_{0}$, proving that the last inequality is in fact strict.

This concludes the proof of Theorem 2.9.

Proof of Lemma 4.2. We investigate the three different cases:

Case 1. For $r \in(0, \sqrt{A / 2 \sqrt{3}}]$ (zone 1 of Fig. 12), since $H_{A, r}$ is admissible and since $d(K) \leqslant 1$ for every convex body $K$, the first equality is obvious, by choosing $K=H_{A, r}$.

Case 2. Let us deal with the zones 2 and 3 of Fig. 12. We first assume that $r \in(0, \sqrt{A / 2 \sqrt{3}}]$ and $D \geqslant \operatorname{Diam}\left(H_{A, r}\right)$. Let $K$ be a maximizer for the problem

$$
\max \{d(K), r(K)=r, \operatorname{Diam}(K)=D,|K|=A\} .
$$

Denoting by $K^{T}$ the smallest convex set tiling the plane and containing $K$, one has

$$
\operatorname{Diam}\left(K^{T}\right) \geqslant D, \quad r\left(K^{T}\right) \geqslant r
$$


Then, by using Theorem 2.7 and by monotonicity of $\left|H^{D, r}\right|$ with respect to $D$ and $r$, we have

$$
\left|K^{T}\right| \geqslant\left|H^{\operatorname{Diam}\left(K^{T}\right), r\left(K^{T}\right)}\right| \geqslant\left|H^{D, r}\right|,
$$

As a consequence, we infer that $d(K)=\frac{|K|}{\left|K^{T}\right|} \leqslant \frac{A}{\left|H^{D, r}\right|}$. Notice that the mapping $A \mapsto \operatorname{Diam}\left(H_{A, r}\right)$ is increasing on its definition set. Using this remark and according to Remark 2.5, since $D \in\left[\operatorname{Diam}\left(H_{A, r}\right), \operatorname{Diam}\left(G_{A, r}\right)\right]$, we have $\left|G^{D, r}\right| \leqslant A \leqslant\left|H^{D, r}\right|$. Moreover, there holds $G^{D, r} \subset H^{D, r}$ by construction. Let us show that $\left(G^{D, r}\right)^{T}=$ $H^{D, r}$. Since

$$
\operatorname{Diam}\left(\left(G^{D, r}\right)^{T}\right) \geqslant D \quad \text { and } \quad r\left(\left(G^{D, r}\right)^{T}\right) \geqslant r
$$

one has $\left|\left(G^{D, r}\right)^{T}\right| \geqslant\left|H^{D, r}\right|$, showing that $\left(G^{D, r}\right)^{T}=H^{D, r}$. Now, consider a convex set $K$ of area $A$ chosen such that $G^{D, r} \subset K \subset H^{D, r}$. Then, since $\left(G^{D, r}\right)^{T}=H^{D, r}$, one has $K^{T}=H^{D, r}$ by continuity and $d(K)=\frac{A}{\left|H^{D, r}\right|}$. Therefore the supremum is reached, whence the conclusion.

Now, assume that

$$
(r, D) \in\left\{(r, D) \mid r>\sqrt{A / 2 \sqrt{3}} \text { and } D \in\left[4 / \sqrt{3} r, \operatorname{Diam}\left(G_{A, r}\right)\right]\right\} .
$$

Then, a convex set $K$ with inradius $r$ and area $A$ cannot be tiling according to Theorem 2.7. Nevertheless, one checks easily that the diameter of the hexagon $H^{D, r}$ is equal to $D$ if, and only if $D \geqslant 4 / \sqrt{3} r$. Therefore, the same argument as below allows to conclude similarly.

Case 3. If $(r, D) \in\left\{(r, D) \mid r>\sqrt{A / 2 \sqrt{3}}\right.$ and $\left.D \leqslant \min \left\{4 / \sqrt{3} r, \operatorname{Diam}\left(G_{A, r}\right)\right\}\right\}$ (zones 4 of Fig. 12), then the diameter of $H^{D, r}$ differs from $D$. Indeed, this is an easy consequence of the first item of Theorem 2.7.

We claim (see below for a proof) moreover that the regular hexagon $H_{r}^{*}$ is the tiling convex set with inradius $r$ and area $A$ having the lowest diameter, or similarly that the regular hexagon $H_{r}^{*}$ is the tiling convex set with inradius $r$ and diameter $D$ having the lowest area.

Let $K$ be a convex set such that $r(K)=r$ and $\operatorname{Diam}(K)=D$, with $(r, D)$ belonging to the zone described above. One has $\operatorname{Diam}\left(K^{T}\right) \geqslant D$ and $r\left(K^{T}\right) \geqslant r$. As a consequence of the claim above, one has necessarily $\operatorname{Diam}\left(K^{T}\right) \geqslant D\left(H_{r\left(K^{T}\right)}^{*}\right)$. Since $K^{T}$ is tiling, one has

$$
\left|K^{T}\right| \geqslant\left|H^{\operatorname{Diam}\left(K^{T}\right), r\left(K^{T}\right)}\right| \geqslant\left|H_{r}^{*}\right| .
$$

according to Theorem 2.7 and the claim above. It follows that for every convex $K$ in the aforementioned zone of the Blaschke diagram, one has $d(K) \leqslant \frac{A}{\left|H_{r}^{*}\right|}$.

Let $K$ be a convex set of area $A$ such that $G^{\operatorname{Diam}\left(K^{T}\right), r\left(K^{T}\right)} \subset K \subset H_{r}^{*}$. We infer from the previous analysis that $K^{T}=H_{r}^{*}$, and $d(K)=A /\left|H_{r}^{*}\right|$, so that it maximizes the density.

To conclude, it remains to prove the claim above. For a given $r>0$, we investigate the problem

$$
\inf \{\operatorname{Diam}(T), T \text { tiling and } r(T) \geqslant r\} \text {. }
$$

Notice first that, by mimicking the arguments used to prove Theorem 2.7, one shows that there exists a solution $T^{*}$ to this problem, and necessarily, $r\left(T^{*}\right)=r$.

Moreover, according to Theorem 2.7, the solution of the more constrained problem

$$
\inf \{\operatorname{Diam}(T), T \text { tiling, } r(T)=r \text { and }|T|=A\},
$$


with $A \geqslant 2 \sqrt{3} r^{2}$, is the $p$-hexagon described in Definition 2.3. Then, by writing

$$
\begin{aligned}
& \inf \{\operatorname{Diam}(T), T \text { tiling and } r(T) \geqslant r\} \\
& \quad=\inf _{A \geqslant 2 \sqrt{3} r^{2}} \inf \{\operatorname{Diam}(T), T \text { tiling, } r(T)=r \text { and }|T|=A\},
\end{aligned}
$$

and using that the area of the $p$-hexagon introduced in Definition 2.3 is an increasing function of the diameter (see Remark 2.8), we infer that $T^{*}$ is such that $\left|T^{*}\right|=2 \sqrt{3} r^{2}$. In other words, $T^{*}=H_{r}^{*}$ and we are done.

5. Conclusion and perspectives. In this paper, we solve several problems in convex geometry, paying attention to the class of plane tiling domains. These problems were motivated by issues in biology related to the shape of eggs of some crustaceans. Of course, the 3D situation is certainly more relevant but a complete mathematical analysis, like in this paper, seems out of range. Nevertheless, some numerical simulations will be done for this problem.

We foresee to investigate a related issue in a forthcoming paper, namely the precise determination of the Blaschke-Santaló diagram, see Figure 12 for the area, diameter and inradius (sometimes known as the $A, D, r$ problem).

Acknowledgement. The first and the third authors were partially supported by the Project "Analysis and simulation of optimal shapes - application to life-sciences" of the Paris City Hall. The authors warmly thank Nicolas Rabet for very stimulating discussions about modelling issues.

\section{Appendix A. Existence of $K^{T}$.}

Since the set of convex bodies contained in a compact $D$ is itself compact for the Hausdorff topology and since the restriction of the Lebesgue measure to this set is continuous [7], it is enough to show that the set of convex tiling domains $T$ with $r(T) \geqslant \varepsilon>0$ is closed for the Hausdorff topology. To prove this claim, let $\left(T_{n}\right)_{n \in \mathbb{N}}$ be a sequence of convex tiling domains converging to $T$. Then $T$ is necessarily convex. Since $T_{n}$ is tiling for every $n$, there exist a sequence $\left(\tau_{n, i}\right)_{i \in \mathbb{N}}$ of affine isometries such that $\mathbb{R}^{2} \subset \bigcup_{i \in \mathbb{N}} \tau_{n, i}\left(T_{n}\right)$, in other words

$$
\forall R>0, \quad D(0, R) \subset \bigcup_{i \in \mathbb{N}} \tau_{n, i}\left(T_{n}\right)
$$

Without loss of generality, we assume that every domain $T_{n}$ contains the origin and the distance of $\tau_{n, i}\left(T_{n}\right)$ (i-th copy of $T_{n}$ ) to the origin is non-decreasing with respect to $i$, for a given $n$.

Let $D=\sup \left(\operatorname{Diam}\left(T_{n}\right)\right), R>0$, and $N=N(R, D, \varepsilon)$ be the minimal number of squares with circumradius $\varepsilon$ to tesselate a disk of radius $R+D$. Then, we claim that

$$
D(0, R) \subset \bigcup_{i=0}^{N} \tau_{n, i}\left(T_{n}\right) \subset D(0, R+2(N+1) D) .
$$

for every $n \in \mathbb{N}$.

Indeed every copy $\tau_{n, i}\left(T_{n}\right)$ contains such a square $C_{i}$, and any copy that contains a point of $D(0, R)$ is necessary included in $D(0, R+D)$, so $C_{i} \subset D(0, R+D)$. Let $K$ be the smallest integer such that $D(0, R) \subset \bigcup_{i=0}^{K} \tau_{n, i}\left(T_{n}\right)$. Then $\bigcup_{i=0}^{K} C_{i}$ is disjoint union of sets included in $D(0, R+D)$. A volume comparison yields $K \leqslant N$ so that 
we have the first inclusion. The second one is straightforward since the distance of $\bigcup_{i=0}^{N} \tau_{n, i}\left(T_{n}\right)$ to the origin cannot be greater than $2(N+1) D$.

To show that $T$ is tilling, let us decompose $\tau_{n, i}$ as $\tau_{n, i}=r_{n, i}+t_{n, i}$ where $r_{n, i}$ is a rotation and $t_{n, i}$ is a translation assimilated (with a slight abuse of notation) to a vector such that $\left\|t_{n, i}\right\| \leqslant R+2(N+1) D$ for all $n \in N$ and $i \leqslant N$. Applying a compactness argument yields the existence of $\tau_{i}$ and $\varphi: \mathbb{N} \mapsto \mathbb{N}$ such that $\tau_{\varphi(n), i} \rightarrow \tau_{i}$ as $n \rightarrow+\infty$. Therefore, one has $\tau_{\varphi(n), i}\left(T_{\varphi(n)}\right) \rightarrow \tau_{i}(T)$ as $n \rightarrow+\infty$. Furthermore, since $\operatorname{int}\left(\tau_{\varphi(n), i}\left(T_{\varphi(n)}\right)\right) \cap \operatorname{int}\left(\tau_{\varphi(n), j}\left(T_{\varphi(n)}\right)\right)=\emptyset$ for $i \neq j$, we get $\operatorname{int}\left(\tau_{i}(T)\right) \cap \operatorname{int}\left(\tau_{j}(T)\right)=\emptyset$ and the sequence $\bigcup_{i=0}^{N} \tau_{\varphi(n), i}\left(T_{\varphi(n)}\right)$ converges to $\bigcup_{i=0}^{N} \tau_{i}(T)$. Finally by stability of the inclusion for the Hausdorf metric, one has $D(0, R) \subset \bigcup_{i=0}^{N} \tau_{i}(T)$.

Using that the last inclusion holds true for every $R>0$, we infer that $T$ is a convex tiling domain.

\section{Appendix B. Proof of Theorem 2.2.}

Let us first consider the case of tiling domains.

Case of tiling domains. Let $K$ be a tiling domain and set $D=\operatorname{Diam}(K)$. There exists a family $\left\{\tau_{i}\right\}_{i \in \mathbb{N}}$ of isometries such that $\mathbb{R}^{2}=\bigcup_{i \in \mathbb{N}} \tau_{i}(K)$. For $R>2 D$, define $P(R)=\bigcup_{\tau_{i}(K) \subset D(0, R)} \tau_{i}(K)$.

Then, by maximality of the diameter, and since $K$ is tiling, one has necessarily $D(0, R-D) \subset P(R)$, and therefore $\sharp\left\{i, \tau_{i}(K) \subset D(0, R)\right\}|K| \geqslant \pi(R-D)^{2}$ and

$$
\frac{2 R}{\sqrt{\sharp\left\{i, \tau_{i}(K) \subset D(0, R)\right\}} \operatorname{Diam}(K)} \leqslant \frac{2 R \sqrt{|K|}}{\sqrt{\pi}(R-D) \operatorname{Diam}(K)} .
$$

Letting $R \rightarrow \infty$, we obtain

$$
\limsup _{R \rightarrow+\infty} \frac{2 R}{\sqrt{\sharp\left\{i, \tau_{i}(K) \subset D(0, R)\right\}} \operatorname{Diam}(K)} \leqslant \frac{2 \sqrt{|K|}}{\sqrt{\pi} \operatorname{Diam}(K)} .
$$

Finally, passing to the infimum over all packings yields

$$
D_{\infty}^{\prime}(K) \leqslant \frac{2 \sqrt{|K|}}{\sqrt{\pi} \operatorname{Diam}(K)}
$$

The conclusion follows by combining this estimate with (2.2).

We now investigate the general case.

General case. In view of proving (2.3), we will use the following result due to Kuperberg's in [9].

Proposition B.1. Every convex set $K \in K$ is contained in a tiling hexagon $K_{\text {kup }}$ satisfying $\left|K_{\text {kup }}\right| /|K| \leqslant 2 / \sqrt{3}$. Moreover, $K_{\text {kup }}$ is a p-hexagon, in other words a hexagon with two opposite parallel sides having the same length ${ }^{5}$.

Let $K \in \mathcal{K}$ and consider the tiling $K_{k u p}$ provided by Proposition B.1. We define a packing of $K$ by placing adequately a copy of $K$ in each cell of $K_{k u p}$. Denoting by

\footnotetext{
${ }^{5}$ Recall that every p-hexagon tiles the plane.
} 
$\left\{\tau_{i}\right\}_{i \in \mathbb{N}}$ the family of isometries used to define this packing, we deduce that

$$
\begin{aligned}
D_{\infty}^{\prime}(K) & \leqslant \limsup _{R \rightarrow \infty} \frac{2 R}{\sqrt{\sharp\left\{i, \tau_{i}\left(K_{\text {kup }}\right) \subset D(0, R)\right\}} \operatorname{Diam}(K)} \\
& =\frac{\operatorname{Diam}\left(K_{\text {kup }}\right)}{\operatorname{Diam}(K)} \limsup _{R \rightarrow \infty} \frac{2 R}{\sqrt{\sharp\left\{i, \tau_{i}\left(K_{\text {kup }}\right) \subset D(0, R)\right\}} \operatorname{Diam}\left(K_{k u p}\right)} \\
& =\frac{2 \sqrt{\left|K_{k u p}\right|}}{\sqrt{\pi} \operatorname{Diam}(K)} \leqslant \sqrt{\frac{2}{\sqrt{3}}} \frac{2 \sqrt{|K|}}{\sqrt{\pi} \operatorname{Diam}(K)},
\end{aligned}
$$

by using the computation above in the case of tiling sets and Proposition B.1.

The expected conclusion follows.

Appendix C. Diameter of $H_{A, r}$ and area of $H^{D, r}$. To avoid any confusion with the notations we will use within this proof, let us denote temporarily by $d$ the diameter of the hexagon $H^{d, r}$ we will consider and by $a$ its area. Let us introduce the points $A, B, C, D$ and $O$, as plotted on Figure 13 .

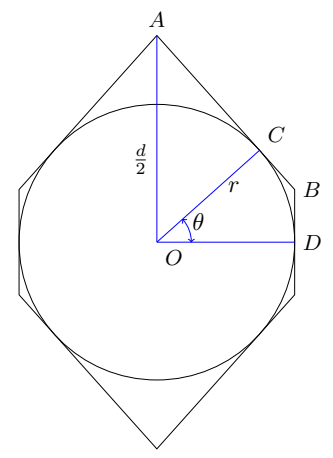

FIG. 13. The hexagon $H^{d, r}$

The area $\left|H^{d, r}\right|$ is equal to 4 times the area of the pentagon $A C B D O$, which is the sum of the area of $A C O$ and the area of $C B D O$, which is twice the area of the triangle $B D O$. Hence, one has $\left|H^{d, r}\right|=4 \times(|A C O|+2|B D O|)$ Let $\theta=\widehat{C O D}$. On has $\sin \theta=2 r / d$. Then, we compute $|A C O|=\frac{d r}{4} \cos \theta=\frac{r}{4} \times \sqrt{d^{2}-4 r^{2}}$. In the orthonormal basis $\left(O ; \frac{\overrightarrow{O D}}{O D}, \frac{\overrightarrow{O A}}{O A}\right)$, the coordinates of $B$ are $\left(r, r \frac{1-\cos (\theta)}{\sin (\theta)}\right)$ and since $\theta=\arcsin (2 r / d)$, we get $|B D O|=\frac{r}{4}\left(d-\sqrt{d^{2}-4 r^{2}}\right)$

Finally, we get that $\left|H^{d, r}\right|=2 r d-r \sqrt{d^{2}-4 r^{2}}$. By inverting the relation $a=$ $2 r d-r \sqrt{d^{2}-4 r^{2}}$ (whenever $a \geqslant 2 \sqrt{3} r^{2}$ and $d \geqslant 2 r$ ), we get that

$$
d=\alpha(a, r)=\frac{1}{3 r}\left(2 a+\sqrt{a^{2}-12 r^{4}}\right),
$$

whence the expression of $\operatorname{Diam}\left(H_{a, r}\right)$ with respect to the parameter $a$.

\section{REFERENCES}


[1] M. Berger. Geometry revealed. Springer, Heidelberg, 2010. A Jacob's ladder to modern higher geometry, Translated from the French by Lester Senechal.

[2] R. Courant. The least dense lattice packing of two-dimensional convex bodies. Comm. Pure Appl. Math., 18:339-343, 1965.

[3] K. R. Doheny. On the lower bound of packing density for convex bodies in the plane. Beiträge Algebra Geom., 36(1):109-117, 1995.

[4] I. Fáry. Sur la densité des réseaux de domaines convexes. Bull. Soc. Math. France, 78:152-161, 1950.

[5] L. Fejes. über die dichteste Kugellagerung. Math. Z., 48:676-684, 1943.

[6] M. Henk and G. A. Tsintsifas. Some inequalities for planar convex figures. Elem. Math., 49(3):120-125, 1994.

[7] A. Henrot and M. Pierre. Shape Variation and Optimization, volume 28 of Tracts in Mathematics. European Mathematical Society, Zürich, 2018.

[8] M. A. Hernández Cifre and G. Salinas. Some optimization problems for planar convex figures. Rend. Circ. Mat. Palermo (2) Suppl., (70, part I):395-405, 2002. IV International Conference in "Stochastic Geometry, Convex Bodies, Empirical Measures \& Applications to Engineering Science", Vol. I (Tropea, 2001).

[9] G. Kuperberg and W. Kuperberg. Double-lattice packings of convex bodies in the plane. Discrete Comput. Geom., 5(4):389-397, 1990.

[10] W. Kuperberg. Packing convex bodies in the plane with density greater than $\frac{3}{4}$. Geom. Dedicata, 13(2):149-155, 1982.

[11] M. Rao. Exhaustive search of convex pentagons which tile the plane. Arxiv.org, arXiv:1708.00274.

[12] C. Zong. Packing, covering and tiling in two-dimensional spaces. Expo. Math., 32(4):297-364, 2014. 